\title{
Approximate Kron reduction methods for electrical networks with applications to plug-and-play control of AC islanded microgrids
}

\author{
Alessandro Floriduz, Member, IEEE, Michele Tucci, Student Member, IEEE, Stefano Riverso, Member, IEEE, and \\ Giancarlo Ferrari-Trecate, Senior Member, IEEE
}

\begin{abstract}
Kron Reduction (KR) is a methodology for analyzing an electrical network by replacing it with a simpler circuit having less nodes but the same terminal behavior of voltages and currents at target vertices. Existing approaches to instantaneous KR, however, can fail in preserving the structure of transfer functions representing power lines. Therefore, even if the original lines are passive $R L C$ circuits, reduced lines might have transfer functions that do not correspond to a physically realizable passive system. To overcome this drawback, in this work we focus on $R L$ line models and propose two approximate $\mathrm{KR}$ algorithms producing reduced lines with first-order transfer functions and capable of representing exactly the asymptotic behavior of electric signals, even if they are unbalanced. Then, we show how to apply these KR methods to the design of decentralized voltage and frequency controllers for AC islanded microgrids. Notably, we focus on the Plug-and-Play (PnP) algorithm previously proposed by some of the authors, which assumes loads connected to the inverter outputs, and generalize it to networks where loads appear in arbitrary positions. Theoretical results are validated with numerical examples and the application of KR for designing PnP controllers is assessed through a simulation on a 21-bus microgrid.
\end{abstract}

Index Terms-Kron reduction, network-reduced model, decentralized control, plug-and-play, AC islanded microgrids.

\section{INTRODUCTION}

Kron Reduction (KR) is standard tool for simplifying linear electrical networks [1] while preserving the behavior of electrical variables at target nodes. KR assumes network nodes are classified either as internal or boundary nodes, and provides an algebraic procedure for computing: (i) the topology of a new network connecting boundary nodes only, (ii) the value of admittances related to new edges and (iii) equivalent currents supplied at boundary nodes accounting for the effect of internal currents in the original network. Graph-theoretical properties of $\mathrm{KR}$ have been analyzed in [2], [3] for DC resistive networks. A general analysis of AC three-phase balanced circuits in Periodic Sinusoidal Steady State (PSSS), termed AC-KR, can be found in [4]. Recently, several studies focused on generalizations of KR methods preserving the electrical behavior of boundary variables not

This work has received support from the Swiss National Science Foundation under the COFLEX project (grant number 200021_169906).

A. Floriduz (alessandro.floriduz@ cern.ch) and M. Tucci (michele.tucci02@universitadipavia.it) are with Dipartimento di Ingegneria Industriale e dell'Informazione, Università degli Studi di Pavia, 27100 Pavia, Italy.

S. Riverso (RiversS@utrc.utc.com) is with United Technologies Research Centre Ireland Ltd., T23 XN53 Cork, Ireland.

G. Ferrari-Trecate (giancarlo.ferraritrecate @epfl.ch) is with Automatic Control Laboratory, École Polytechnique Fédérale de Lausanne (EPFL), 1015 Lausanne, Switzerland.

giancarlo.ferraritrecate@epfl.ch, Corresponding author. only in PSSS, but also during transients [2], [4], [5], [6]. In these instantaneous KR procedures, network line admittances are replaced by differential models, and sufficient conditions guaranteeing well-posedness of the network reduction process have been studied.

It should be noted that KR is not the only approach for obtaining dynamical models of reduced networks. For instance, in the power system literature, multiport equivalencing methods based on the idea of fitting reduced models on samples of the network frequency response have been proposed [7]. However, KR is different because it provides a mathematical framework for simplifying electric networks without approximating the time evolution of boundary variables.

Existing instantaneous KR methods have a relevant limitation: they do not guarantee that reduced line models will have the same structure of the original ones, e.g. simple $R L$ lines could result in reduced lines with more complex dynamics [6]. Therefore, even though this does not happen for simple cases such as DC resistive or homogeneous networks [4], [3], [2], in general one faces the problem of devising approximate instantaneous KR methods for preserving selected features of line models.

Motivated by applications to low-voltage microgrids [8], in this paper we focus on $R L$ line models. Indeed, wires in microgrids have usually a short length and capacitive effects can be disregarded, see [9, Chapter 5], [10, Chapter 6] and [11, p. 259]. For this kind of lines, we propose two novel approximate KR methods termed approximate AC Kron Reduction (aAC-KR) and hybrid Kron Reduction (hKR). We show that these techniques ensure asymptotic equivalence between original and reduced models, if networks reach a PSSS. Unlike the instantaneous KR procedures in [2], [4], [5], [6], aAC-KR and hKR provide reduced circuits whose lines have first-order transfer functions, as the original lines. The features of our methods are then validated through numerical simulations.

In order to demonstrate the practical relevance of aAC-KR and hKR, in the second part of the paper we show how to use them to design decentralized voltage and frequency controllers for AC microgrids, i.e. electrical networks composed of Distributed Generation Units (DGUs) and loads, operating either connected or isolated from the main grid [8], [12], [13], [14], [15], [16]. In particular, we focus on the latter operation mode, thus dealing with Islanded microGrids (ImGs). KR has already found applications to ImGs; for instance, in [17], [18] KR is advocated as the procedure for mapping ImGs with general topologies into their equivalent load-connected circuits, where loads appear only at the terminals of inverters. This can be 
done by labeling other load nodes as internal and applying $\mathrm{KR}$ to eliminate them. Following this procedure, any control design method for load-connected ImG could be directly extended to ImGs with arbitrary topologies by performing control synthesis on the reduced network. However, control schemes for ImGs often rely on additional assumptions, such as specific line models. For instance, droop controllers are tailored to either mainly inductive or mainly resistive lines [19], while Plug-and-Play (PnP) controllers in [20] assume $R L$ lines. In this paper, we focus on the latter class of decentralized regulators, as they enjoy the following features: (i) when a DGU is plugged in, the design of the local controller can be done on the fly as it requires no global model but only the parameters of power lines connected to the DGU; (ii) one can test in an automatic way if the addition/removal of a DGU is critical for ImG stability by solving a local optimization problem. Within this framework, we show that both aAC-KR and hKR can be used to extend the design method in [20] (which assumes load-connected ImGs and $R L$ lines) to arbitrary interconnections of DGUs and loads, while preserving the structure of transfer functions describing power lines. The design of PnP controllers based on the proposed approximate KR methods is tested on a 21-bus ImG derived from the IEEE test feeder in [21], enhanced with switches yielding changes of line topology and plug-in/out of DGUs. Simulations performed in PSCAD confirm the applicability of our methods to control of ImGs.

The paper is structured as follows. Section II summarizes the existing KR methods, highlighting the corresponding features and limitations. In Section III, we present the approximate KR methods (aAC-KR and hKR), and in Section IV we assess their performances through numerical simulations. In Section V, we (i) introduce the models of DGUs and lines, (ii) review the results in [20] about PnP control design for load-connected AC ImGs, and (iii) show how to combine this methodology with the proposed approximate KR techniques. Simulations illustrating the joint use of aAC-KR/hKR and PnP design are given in Section VI. Finally, Section VII concludes the paper.

A preliminary version of this work has been presented at the 15th European Control Conference [22]. Differently from [22], in which only hKR has been described, in the present paper we (i) introduce aAC-KR, (ii) perform a detailed comparison between the two approximate KR methods including numerical examples and simulations, (iii) provide a more general and rigorous proof of [22, Proposition 1] showing the asymptotic equivalence between the original network and the reduced circuit obtained through hKR, and (iv) extend this latter result also to aAC-KR.

Notation and basic definitions. We use $f^{a b c}(t)=$ $\left[f_{a}(t), f_{b}(t), f_{c}(t)\right]^{T} \in \mathbb{R}^{3}$ for denoting three-phase signals in the $a b c$ frame. To $f^{a b c}(t)$, we associate its representation in the $d q 0$ reference frame (i.e. $\left.f^{d q 0}(t)\right)$. It is obtained from $f^{a b c}(t)$ through the Park transformation [23], denoted with $T(\theta(t))$, $\theta(t)=\omega_{0} t, \omega_{0}$ being the nominal network angular frequency. To three-phase signals without zero-sequence component (i.e. $f^{0}(t)=0$ ) we can associate their complex $d q$-representation $f^{d q}(t)=f^{d}(t)+$ i $f^{q}(t)$ without loss of generality.
When clear from the context, we omit time dependence of electrical quantities. $\mathscr{L}[\cdot]$ identifies the Laplace-transform operator. Moreover, for a real valued function $f(t)$, we indicate with $\mathcal{H}[f(t)]$ and $\mathscr{A}[f(t)]$ the corresponding Hilbert transform and analytic signal, respectively. They are defined as follows.

Definition 1 (Hilbert transform). The Hilbert transform $\mathcal{H}$ of a real function $f(t)$ is defined as [24]:

$$
\mathcal{H}(f(t))(t)=\text { p.v. } \int_{-\infty}^{\infty} f(\tau) h(t-\tau) d \tau=\frac{1}{\pi} \text { p.v. } \int_{-\infty}^{\infty} \frac{f(\tau)}{t-\tau} d \tau
$$

where $h(t)=1 /(\pi t)$ and p.v. denotes the Cauchy principal value (since the integral is improper).

Definition 2 (Analytic signal). The analytic signal $\bar{f}(t)=$ $\mathscr{A}[f(t)]$ is a complex-valued function, whose real part is equal to $f(t)$, and its imaginary part is $\mathcal{H}[f(t)]$. In formulae [24]:

$$
\bar{f}(t)=\mathscr{A}[f(t)]=f(t)+\mathrm{i} \mathcal{H}[f(t)] .
$$

The analytic signal generalizes the phasor concept. Notably, the latter one is restricted to time-invariant amplitude, phase and frequency, while the analytic signal allows for timevarying parameters [24].

Algebraic graph theory. The cardinality of the finite set $S$ will be denoted with $|S|$. According to the definitions in [25], a weighted graph $\mathcal{G}=(\mathcal{V}, \mathcal{E}, W)$ of order $n=|\mathcal{V}|$ is given by a finite set of nodes $\mathcal{V}=\{1, \ldots, n\}$, a set of edges $\mathcal{E} \subseteq \mathcal{V} \times \mathcal{V}$ and a diagonal matrix $W$ of dimension $|\mathcal{E}| \times|\mathcal{E}|$, collecting on its diagonal weights $W_{e}, e \in \mathcal{E}$. In this work, weights can be real numbers, complex numbers or SISO transfer functions (in this case, we replace $W$ with $W(s)$ ). An edge $e \in \mathcal{E}$ is a self-loop if $e=(i, i)$, for some $i \in \mathcal{V}$; all graphs in this work will not contain self-loops. A graph is undirected if $(x, y) \in$ $\mathcal{E} \Longrightarrow(y, x) \in \mathcal{E}$. In this case, the pairs $(x, y)$ and $(y, x)$ are considered as identical and unordered. Otherwise, the graph is said to be directed. The set of neighbors of node $i \in \mathcal{V}$ is $\mathcal{N}_{i}=$ $\{j:(i, j) \in \mathcal{E}$ or $(j, i) \in \mathcal{E}\}$. An undirected graph is connected if, for all $(i, j) \in \mathcal{V} \times \mathcal{V}$, there is a path from $i$ to $j$. A directed graph is weakly connected if its undirected version (obtained by neglecting the edge orientation) is connected. The incidence matrix of $\mathcal{G}[25]$ is denoted with $B \in\{-1,0,1\}^{|\mathcal{V}| \times|\mathcal{E}|}$. The Laplacian of $\mathcal{G}$ is defined as the matrix $\mathbb{L}=B \cdot W \cdot B^{T}$ [25]. Note that, by construction, the Laplacian matrix has zero row sum. We recall that, to a Laplacian matrix is possible to associate a unique graph, up to the orientation of edges.

\section{KRON REDUCTION METHODS FOR ELECTRICAL NETWORKS}

Let $\mathcal{G}=(\mathcal{V}, \mathcal{E}, W(s))$ be the weighted directed graph associated with a given circuit. Following the formalism introduced in [2], [4], [5], [26], each vertex corresponds to a node of the network and each edge is a branch of the circuit (see the example in Figure 1). For performing $\mathrm{KR}$, the vertex set $\mathcal{V}$ is partitioned into a set of boundary nodes $\mathcal{V}_{b}$ and a set of internal nodes $\mathcal{V}_{\ell}$. More specifically, subset $\mathcal{V}_{b}$ identifies the nodes at which the behavior of electrical variables must be preserved, while $\mathcal{V}_{\ell}$ contains the nodes to be eliminated.

We denote the nodal currents injected at boundary nodes with $I_{b}$; the nodal currents injected at internal nodes are 
$I_{\ell}$ and they are positive if entering into nodes. Nodal voltages $V$ are partitioned analogously. In this work, each edge $e_{1}, \ldots, e_{|\mathcal{E}|} \in \mathcal{E}$ corresponds to an electric $R L$ line and the orientation of the edges is arbitrary. We adopt the following sign convention: reference directions of line currents coincide with edges orientations and line voltages $V_{e}, e=(i, j) \in \mathcal{E}$ are defined as $V_{i}-V_{j}$. Moreover, the weight of every edge is given by its admittance transfer function $W_{e}(s)$ accounting for the dynamics of line $e \in \mathcal{E}$.

Let us now consider an electrical network composed of $n>$ 0 nodes, partitioned into $n_{b}>0$ boundary nodes and $n_{\ell}=$ $n-n_{b}>0$ internal nodes. We collect the nodal currents in the following vector: $I=\left[I_{b}^{T}, I_{\ell}^{T}\right]^{T}$, where $I_{b}=\left[I_{b_{1}}, \ldots, I_{b_{n_{b}}}\right]^{T}$ and $I_{\ell}=\left[I_{\ell_{1}}, \ldots, I_{\ell_{n_{\ell}}}\right]^{T}$. Nodal voltages $V=\left[V_{b}^{T}, V_{\ell}^{T}\right]^{T}$ are partitioned analogously. In order to account for the network interconnections, by applying Kirchhoff's Current Law (KCL) and Kirchhoff's Voltage Law (KVL) one obtains [6]:

$$
\left(\begin{array}{l}
I_{b}(s) \\
I_{\ell}(s)
\end{array}\right)=\left(\begin{array}{ll}
\mathbb{L}_{b b}(s) & \mathbb{L}_{b \ell}(s) \\
\mathbb{L}_{\ell b}(s) & \mathbb{L}_{\ell \ell}(s)
\end{array}\right)\left(\begin{array}{l}
V_{b}(s) \\
V_{\ell}(s)
\end{array}\right)
$$

that is $I(s)=\mathbb{L}(s) V(s)$, where $\mathbb{L}(s)$ is the graph Laplacian of the graph $\mathcal{G}$ with weights

$$
W_{i j}(s)=\frac{1}{R_{i j}+s L_{i j}}
$$

modeling the admittance transfer function associated with edges $(i, j) \in \mathcal{E}$. By construction, one has $\mathbb{L}_{i j}(s)=-W_{i j}(s)$ if $(i, j) \in \mathcal{E}$ and $\mathbb{L}_{i i}(s)=-\sum_{j \in \mathcal{N}_{i}} \mathbb{L}_{i j}(s)$.

At this point, we have all the elements for introducing KR. In particular, in the sequel we will focus on summarizing two existing approaches, namely KR in phasors domain [4] (called hereafter $\mathrm{AC}-\mathrm{KR}$ ) and instantaneous KR [6].

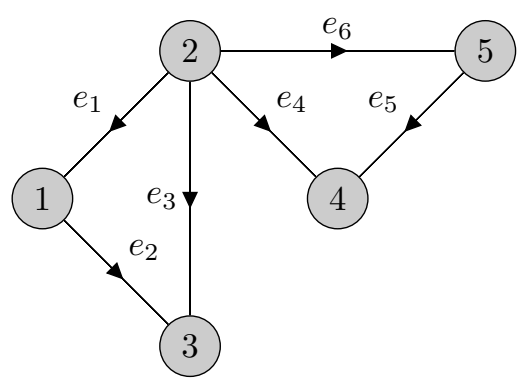

Fig. 1: Graph representing an electrical network.

\section{A. $A C-K R$}

Let us consider the directed graph $\mathcal{G}=(\mathcal{V}, \mathcal{E}, W(s))$, where transfer functions $W_{i j}(s)$ in the form (2) represent the relation between $\mathscr{L}\left[V_{i}(t)-V_{j}(t)\right]$ and $\mathscr{L}\left[I_{i j}(t)\right]$. We assume the network is in PSSS with angular frequency $\omega_{0}$, hence $V_{i}(t)=A_{i} \cos \left(\omega_{0} t+\phi_{i}\right)$ and $I_{i}(t)=B_{i} \cos \left(\omega_{0} t+\gamma_{i}\right), \forall i \in \mathcal{V}$. Moreover, we can associate each cosinusoid $V_{i}(t)$ with the corresponding rotating phasor $\vec{V}_{i}=A_{i} \exp \left(\mathrm{i}\left(\omega_{0} t+\phi_{i}\right)\right)$ [27]. Current phasors $\vec{I}_{i}$ are defined analogously. Let us now define vectors $\vec{V}=\left[\vec{V}_{1}, \vec{V}_{2}, \ldots, \vec{V}_{n}\right]^{T}, \vec{I}=\left[\vec{I}_{1}, \vec{I}_{2}, \ldots, \vec{I}_{n}\right]^{T}$, and the impedance $Z_{i j}=R_{i j}+\mathrm{i} \omega_{0} L_{i j}$ of line $(i, j)$. The relation between nodal currents and nodal voltages is then given by:

$$
\vec{I}=\mathbb{L}^{A C} \cdot \vec{V}
$$

where $\mathbb{L}_{i j}^{A C}=-1 / Z_{i j}$, if $(i, j) \in \mathcal{E}$, and $\mathbb{L}_{i i}^{A C}=$ $\sum_{j \in \mathcal{N}_{i}} 1 / Z_{i j}$. In particular, by construction, $\mathbb{L}^{A C}=\mathbb{L}\left(\mathrm{i} \omega_{0}\right)$, with $\mathbb{L}$ defined in (1).

Definition 3 (AC-KR). Let $\vec{V}, \vec{I}$ and $\mathbb{L}^{A C}$ be partitioned into boundary and internal components as in (1) and assume $\mathbb{L}_{\ell \ell}^{A C}$ is invertible. $A C-K R$ is given by the graph $\mathcal{G}_{\text {red }}^{A C}=$ $\left(\mathcal{V}_{b}, \mathcal{E}_{\text {red }}, W_{\text {red }}^{A C}\right)$ associated with the reduced Laplacian

$$
\mathbb{L}_{\text {red }}^{A C}=\mathcal{K}\left(\mathbb{L}^{A C}\right)=\mathbb{L}_{b b}^{A C}-\mathbb{L}_{b \ell}^{A C}\left(\mathbb{L}_{\ell \ell}^{A C}\right)^{-1} \mathbb{L}_{\ell b}^{A C},
$$

and

$$
\begin{gathered}
\overrightarrow{I_{b}}=\mathbb{L}_{\text {red }}^{A C} \vec{V}_{b}-\mathcal{T}^{A C} \vec{I}_{\ell} \\
\mathcal{T}^{A C}=-\mathbb{L}_{b \ell}^{A C}\left(\mathbb{L}_{\ell \ell}^{A C}\right)^{-1},
\end{gathered}
$$

where $\mathcal{K}(\cdot)$ denotes the KR operator and $\mathcal{T}^{A C}$ is the accompanying matrix of $\mathbb{L}^{A C}$.

Conditions for the invertibility of $\mathbb{L}_{\ell \ell}^{A C}$ are given in the next Lemma, which has been adapted from [28, Lemma 2.4.20].

Lemma 1. If all lines are represented by $R L$ models and the graph $\mathcal{G}$ is weakly connected, then the matrix $\mathbb{L}_{\ell \ell}^{A C}$ is nonsingular.

It can be shown that the matrix $\mathbb{L}_{r e d}^{A C}$ in (4a)-(4b) is still the Laplacian of the Kron reduced graph $\mathcal{G}_{\text {red }}^{A C}$ [4] that is uniquely defined up to the orientation of edges (which can be arbitrarily chosen). Moreover, $W_{r e d, i j}^{A C}=-\left(\mathbb{L}_{r e d}^{A C}\right)_{i j}$ for $(i, j) \in \mathcal{E}_{r e d}$, while $\mathcal{T}^{A C}$ provides the vector of equivalent nodal currents

$$
\tilde{\vec{I}}_{b}=\mathcal{T}^{A C} \vec{I}_{\ell}
$$

to be injected at boundary nodes of $\mathcal{G}_{\text {red }}^{A C}$ in order to account for the effect of eliminated currents $\vec{I}_{\ell}$.

It can be shown that, as long as one provides the same voltages $\vec{V}_{b}$ in $\mathcal{G}$ and $\mathcal{G}_{\text {red }}^{A C}$, the same current absorption $I_{b}$ is obtained in both cases, if currents (5) are injected into boundary nodes $\mathcal{G}_{\text {red }}^{A C}$ [3], [4].

At this point, it is convenient to show, through a simple example, how to perform AC-KR, hence computing $\mathbb{L}^{A C}$, $\mathbb{L}_{\text {red }}^{A C}$ and $\mathcal{T}^{A C}$, defined in (3), (4a) and (4c), respectively.

Example 1. We consider the linear, single-phase circuit $\mathcal{G}$ shown in Figure 2a, and we assume the network is PSSS with angular frequency $\omega_{0}=2 \pi f_{0}, f_{0}=50 \mathrm{~Hz}$. Consequently, all the voltages and currents can be represented through their associated rotating phasors, i.e. $\vec{V}=\left[\vec{V}_{1}, \vec{V}_{2}, \vec{V}_{3}, \vec{V}_{4}\right]^{T}, \vec{I}=$ $\left[\vec{I}_{1}, \vec{I}_{2}, \vec{I}_{3}, \vec{I}_{4}\right]^{T}$. The vertex set of $\mathcal{G}$ is partitioned in boundary and internal nodes as follows: $\mathcal{V}=\left\{\mathcal{V}_{b}, \mathcal{V}_{\ell}\right\}$, where $\mathcal{V}_{b}=$ $\{1,2,3\}$ and $\mathcal{V}_{\ell}=\{4\}$. As regards the edges $e_{1}, e_{2}, e_{3} \in$ $\mathcal{E}$, they correspond to $R L$ power lines, and their parameters are reported in Table I. Our aim is to derive, via $A C-K R$, the equivalent network $\mathcal{G}_{\text {red }}^{A C}$ connecting boundary nodes only (see Figure 2b). Notice that this operation amounts to a $Y-\Delta$ 
transformation. We start by writing the incidence and weights matrices of $\mathcal{G}$ :

$$
\begin{aligned}
B & =\left(\begin{array}{ccc}
-1 & 0 & 0 \\
0 & -1 & 0 \\
0 & 0 & -1 \\
1 & 1 & 1
\end{array}\right), \\
W^{A C} & =\left(\begin{array}{ccc}
W_{14}^{A C} & 0 & 0 \\
0 & W_{24}^{A C} & 0 \\
0 & 0 & W_{34}^{A C}
\end{array}\right),
\end{aligned}
$$

where

$$
\begin{aligned}
& W_{14}^{A C}=\frac{1}{Z_{14}}=0.247-1.552 \mathrm{i} \\
& W_{24}^{A C}=\frac{1}{Z_{24}}=0.041-0.451 \mathrm{i} \\
& W_{34}^{A C}=\frac{1}{Z_{34}}=0.092-0.290 \mathrm{i}
\end{aligned}
$$

Then, we compute the Laplacian matrix $\mathbb{L}^{A C}=B \cdot W^{A C} \cdot B^{T}$, and partition it as in (1), thus having:

$$
\mathbb{L}^{A C}=\left(\begin{array}{ll}
\mathbb{L}_{b b}^{A C} & \mathbb{L}_{b \ell}^{A C} \\
\mathbb{L}_{\ell b}^{A C} & \mathbb{L}_{\ell \ell}^{A C}
\end{array}\right),
$$

with

$$
\begin{gathered}
\mathbb{L}_{b b}^{A C}=\operatorname{diag}(0.247-1.552 \mathrm{i}, 0.041-0.451 \mathrm{i}, 0.092-0.289 \mathrm{i}), \\
\mathbb{L}_{b \ell}^{A C}=\left(\begin{array}{c}
-0.247+1.552 \mathrm{i} \\
-0.041+0.451 \mathrm{i} \\
-0.092+0.289 \mathrm{i}
\end{array}\right), \quad \mathbb{L}_{\ell b}^{A C}=\left(\mathbb{L}_{b \ell}^{A C}\right)^{T},
\end{gathered}
$$

and

$$
\mathbb{L}_{\ell \ell}^{A C}=0.380-2.292 \mathrm{i}
$$

Next, using (4a), one obtains the reduced Laplacian $\mathbb{L}_{\text {red }}^{A C}=$

$$
\left(\begin{array}{ccc}
0.087-0.501 \mathrm{i} & -0.026+0.305 \mathrm{i} & -0.061+0.196 \mathrm{i} \\
-0.026+0.305 \mathrm{i} & 0.039-0.363 \mathrm{i} & -0.014+0.057 \mathrm{i} \\
-0.061+0.196 \mathrm{i} & -0.014+0.057 \mathrm{i} & 0.075-0.253 \mathrm{i}
\end{array}\right)
$$

while the accompanying matrix $\mathcal{T}^{A C}$, is given by (4c), as

$$
\mathcal{T}^{A C}=\left(\begin{array}{c}
0.676-0.004 \mathrm{i} \\
0.194-0.014 \mathrm{i} \\
0.129+0.019 \mathrm{i}
\end{array}\right) .
$$

At this point, we have all the elements for computing the weights of the reduced edges $r_{1}, r_{2}, r_{3} \in \mathcal{E}_{\text {red }}$ as $W_{\text {red,ij }}^{A C}=$ - $\left(\mathbb{L}_{r e d}^{A C}\right)_{i j}$ (see Table I for the corresponding line parameters). Moreover, from (5) the equivalent vector of currents injected at boundary nodes of $\mathcal{G}_{\text {red }}^{A C}$ is

$$
\tilde{\overrightarrow{I_{b}}}=\mathcal{T}^{A C} \vec{I}_{4} \text {. }
$$

In the next Section, we summarize a KR method which, differently from AC-KR, allows to reduce linear circuits while preserving the behavior of boundary variables also in nonstationary regime (e.g. during transients).

\begin{tabular}{ccc}
\hline Edge & Resistance $[\Omega]$ & Inductance $[\mathrm{mH}]$ \\
\hline$e_{1}$ & 0.1 & 2 \\
$e_{2}$ & 0.2 & 7 \\
$e_{3}$ & 1 & 10 \\
\hline$r_{1}$ & 0.2746 & 10.354 \\
$r_{2}$ & 1.4482 & 14.8132 \\
$r_{3}$ & 3.9315 & 52.3706 \\
\hline
\end{tabular}

TABLE I: Example 1 - Line parameters of the original and reduced network.

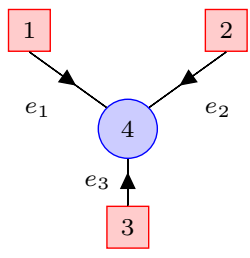

(a) Original network $\mathcal{G}$.

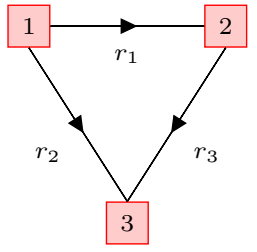

(b) Reduced network $\mathcal{G}_{\text {red }}^{A C}$.
Fig. 2: Example 1 - Original and reduced networks. Boundary and interior nodes are represented by red squares and blue circles, respectively.

\section{B. Instantaneous $K R$}

Instantaneous $\mathrm{KR}$ is a general reduction method which can be applied to any system composed of passive $R, L$, $C$ components while guaranteeing the equivalence between original and reduced boundary variables not necessarily in PSSS [6]. This method, however, presents some drawbacks (discussed later in this Section) which further motivate the need for the approximated KR methods proposed in Section III.

Definition 4 (Instantaneous KR). Assume $\mathbb{L}_{\ell \ell}(s)$ in (1) is invertible 1 for some $s \in \mathbb{C}$. Instantaneous $K R$ is given by the graph $\mathcal{G}_{\text {red }}=\left(\mathcal{V}_{b}, \mathcal{E}_{\text {red }}, W_{\text {red }}(s)\right)$ associated with $\mathbb{L}_{\text {red }}(s)=\mathcal{K}(\mathbb{L}(s)$ ) (up to the orientation of edges, which can be arbitrarily chosen) and [6]

$$
\begin{gathered}
I_{b}(s)=\mathbb{L}_{r e d}(s) V_{b}(s)-\mathcal{T}(s) I_{\ell}(s) \\
\mathcal{T}(s)=-\mathbb{L}_{b \ell}(s) \mathbb{L}_{\ell \ell}^{-1}(s) .
\end{gathered}
$$

We highlight that (9b) corresponds to (4c) when $\mathbb{L}^{A C}$ is replaced by $\mathbb{L}(s)$. Moreover, (9a) provides a relation similar to (4b). In particular, the vector

$$
\tilde{I}_{b}(s)=\mathcal{T}(s) I_{\ell}(s)
$$

identifies the equivalent currents to be injected into the boundary nodes. We further notice that, by construction, for given internal currents $I_{\ell}(t)$ and voltages $V_{b}(t), t \geq 0$ (assuming null initial conditions), currents $I_{b}(t)$ computed through (1) and (9a) are identical at all times (hence the name instantaneous $\mathrm{KR})$.

Remark 1. Instantaneous KR has some limitations. More specifically, the branch admittances of the reduced network might have different dynamics with respect to those in the

\footnotetext{
${ }^{1}$ Conditions for the invertibility of $\mathbb{L}_{\ell \ell}(s)$ have been studied in [6].
} 
original circuit and, what is worse, might not even represent a physical circuit built with passive $R, L, C$ elements. Consequently, a key issue is to understand when weights $W_{\text {red,ij }}(s)$ can be written as in (2) replacing $R_{i j}$ and $L_{i j}$ with suitable parameters $\tilde{R}_{i j}$ and $\tilde{L}_{i j}$. It has been shown that this is guaranteed only under special assumptions, for instance if original lines are homogeneous [4], i.e. $\frac{R_{e_{i}}}{L_{e_{i}}}=\frac{R_{e_{j}}}{L_{e_{j}}}, \forall e_{i}, e_{j} \in \mathcal{E}$. In this case, one also has $\tilde{R}_{i j}>0$ and $\tilde{L}_{i j}>0$.

\section{APPROXIMATE KR METHODS}

In this Section, we propose two approximated KR approaches, termed approximate $A C-K R$ (aAC-KR) and hybrid $K R$ (hKR), respectively. Both the methods allow to (i) extend the application of AC-KR to electrical variables not necessarily in PSSS, and (ii) overcome the realization problems of instantaneous KR discussed in Remark 1 (at least, as long as the parameters of the reduced circuit are positive). In particular, the proposed approximate techniques provide reduced circuits whose lines have the same $R L$ "nature" as the original ones.

From a practical point of view, aAC-KR and hKR share the idea of using the line parameters (resistances and inductances) obtained from AC-KR at frequency $\omega_{0}$, while differ in the way equivalent internal currents referred to boundary nodes are computed.

In the sequel, we first define the approximated Kron reduced graph; it is exploited in both aAC-KR and hKR for preserving the $R L$ nature of the reduced lines. Then, we separately characterize the proposed methods by describing how the equivalent boundary currents (accounting for the effects of eliminated internal currents) are computed. Finally, in Proposition 1, we prove that both aAC-KR and hKR ensure asymptotic equivalence between original and reduced models if the considered network reaches a PSSS.

Definition 5 (Approximate Kron reduced graph). The approximate Kron reduced graph $\mathcal{G}_{\text {red }}^{\mathcal{A}}$ of the original network $\mathcal{G}$ with respect to a specified angular frequency $\omega_{0}$ is obtained by:

1) computing $\mathbb{L}_{\text {red }}^{A C}=\mathcal{K}\left(\mathbb{L}^{A C}\right)$ and the associated directed graph $\mathcal{G}_{\text {red }}^{A C}=\left(\mathcal{V}_{b}, \mathcal{E}_{\text {red }}, W_{\text {red }}^{A C}\right)$, at the given $\omega_{0}$;

2) setting $\mathcal{G}_{\text {red }}^{\mathcal{A}}=\left(\mathcal{V}_{b}, \mathcal{E}_{\text {red }}, W_{\text {red }}^{\mathcal{A}}(s)\right)$ where, for $(i, j) \in$ $\mathcal{E}_{\text {red }}$

$$
\begin{gathered}
W_{r e d, i j}^{\mathcal{A}}(s)=\frac{1}{\tilde{R}_{i j}+s \tilde{L}_{i j}}, \\
\tilde{Z}_{i j}=-\left(\mathbb{L}_{r e d, i j}^{A C}\right)^{-1}, \tilde{R}_{i j}=\operatorname{Re}\left(\tilde{Z}_{i j}\right), \tilde{L}_{i j}=\frac{1}{\omega_{0}} \operatorname{Im}\left(\tilde{Z}_{i j}\right) .
\end{gathered}
$$

Notice that, in $\mathcal{G}_{\text {red }}^{\mathcal{A}}$ line impedances have still the dynamics (2) but resistances $\tilde{R}_{i j}$ and inductances $\tilde{L}_{i j}$ are those predicted by $\mathrm{AC}-\mathrm{KR}$ at angular frequency $\omega_{0}$. This is a key difference from instantaneous KR (see Remark 1). Moreover, this choice of the network weights will allow us to describe, without introducing any approximation, the asymptotic behavior of the network when input variables are in PSSS with angular frequency $\omega_{0}$ (see Proposition 1 in Section III-C). Note also that, by construction, the graphs associated with $\mathbb{L}_{\text {red }}^{A C}$ and
$\mathbb{L}_{\text {red }}(s)$ have the same set of undirected edges. Therefore, by choosing the same orientation, the set of directed edges of $\mathcal{G}_{\text {red }}$ and $\mathcal{G}_{\text {red }}^{A C}$ can be made identical (this is why they have been both denoted with $\mathcal{E}_{\text {red }}$ ).

Remark 2. AC-KR does not guarantee positivity of the reduced resistances and inductances in (11b). Based on the results of [4], one expects that negative values can occur if the time constants of the original lines are spread in a wide range. In practical applications such as microgrids, however, electrical lines are usually similar and so are their time constants.

\section{A. Approximate AC-KR}

The idea behind aAC-KR is to use the accompanying matrix $\mathcal{T}^{A C}$ in (4c), computed with respect to $\omega_{0}$, to obtain an approximation of the equivalent internal currents referred to boundary nodes when the network is in non-stationary regime. Since, by construction, $\mathcal{T}^{A C}$ is a complex valued matrix (to be multiplied by phasors $\vec{I}_{\ell}$ so as to give other phasors, as shown in (10)), we must now find a way to represent non-sinusoidal internal currents $I_{\ell}(t)$ through complex functions.

In general, given a real-valued function $f(t)$, there is not a unique way to represent it though a complex function $\tilde{f}(t)$, such that $\operatorname{Re}(\tilde{f}(t))=f(t)$ [29]. However, from e.g. [29], one has that, among all these possible complex functions, the analytic signal $\bar{f}(t)=\mathscr{A}(f(t))$ is the only representation which guarantees harmonic correspondence (i.e. if $f(t)$ becomes a sinusoid, then $\bar{f}(t)$ coincides with the corresponding phasor $\vec{f})$ and simultaneously verifies the generalized Tellegen's theorem [30]. For this reason, the first approximate KR method we propose exploits the notion of analytic signal of the real function $I_{\ell}(t)$, and it is defined as follows.

Definition 6 (aAC-KR). Let $\bar{J}_{\ell}(t)=\mathscr{A}\left(I_{\ell}(t)\right)$ be a complex function denoting the analytic signal of the real function $I_{\ell}(t)$, and let $\operatorname{Re}\left(\mathcal{T}^{A C} \bar{J}_{\ell}(t)\right)$ indicate the approximated internal currents to be injected into the boundary nodes of the reduced network. aAC-KR is given by the network $\mathcal{G}_{\text {red }}^{\mathcal{A}}$ and the relation

$$
I_{b}(s)=\mathbb{L}_{r e d}^{\mathcal{A}}(s) V_{b}(s)-\tilde{J}_{b}(s),
$$

where $\mathbb{L}_{\text {red }}^{\mathcal{A}}(s)$ is the Laplacian of $\mathcal{G}_{\text {red }}^{\mathcal{A}}$ and

$$
\tilde{J}_{b}(s)=\mathscr{L}\left[\operatorname{Re}\left(\mathcal{T}^{A C} \bar{J}_{\ell}(t)\right)\right] .
$$

From (12b), we notice that the interior currents reduction is performed using the entries of $\mathcal{T}^{A C}$, which, by definition, refer to variables in PSSS. Therefore, we expect the aAC-KR to lose accuracy when applied to nonlinear circuits or networks at a frequency $\omega \neq \omega_{0}$ (where $\omega_{0}$ is used to compute $\mathcal{G}_{\text {red }}^{\mathcal{A}}$ and $\mathcal{T}^{A C}$ ). To overcome this issue, in the sequel we present a more sophisticated approximate KR method.

\section{B. Hybrid Kron Reduction}

Differently from aAC-KR, in hKR the mapped internal currents are the same as in (10), as described in the following. 
Definition 7 (hKR). $h K R$ is given by the network $\mathcal{G}_{\text {red }}^{\mathcal{A}}$ and the relation

$$
I_{b}(s)=\mathbb{L}_{r e d}^{\mathcal{A}}(s) V_{b}(s)-\tilde{I}_{b}(s)
$$

where $\mathbb{L}_{\text {red }}^{\mathcal{A}}(s)$ is the Laplacian of $\mathcal{G}_{\text {red }}^{\mathcal{A}}$ and $\tilde{I}_{b}(s)$ is defined in (10).

In Section IV, we will show that, compared to aAC-KR, hKR provides more accurate approximations of the boundary electrical variables; this is due to the fact that, with hKR, internal currents are mapped into boundary ones with no approximations (currents $I_{b}$ in (13) are the same as currents $\tilde{I}_{b}$ in (10)). On the other hand, performing the interior currents reduction using the entries of $\mathcal{T}(s)$ is more complex than implementing (12). In fact, the transfer functions of $\mathcal{T}(s)$ may have a high degree, in the case of large networks, and this could be an implementation limit.

Example 2. We want to apply (separately) $a A C-K R$ and $h K R$ to the circuit described in Example 1 (Figure 2a), assuming that electric variables are not necessarily in PSSS.

The incidence matrix $B$ has the form (6), while the weights matrix $W(s)$ is:

$$
W(s)=\left(\begin{array}{ccc}
\frac{10}{1+0.02 s} & 0 & 0 \\
0 & \frac{5}{1+0.035 s} & 0 \\
0 & 0 & \frac{1}{1+0.01 s}
\end{array}\right)
$$

where resistances and inductances of the weights in $W(s)$ are the same of Example 1. It follows that, at PSSS with $\omega_{0}=$ $2 \pi 50 \mathrm{rad} / \mathrm{s}, W\left(\mathrm{i} \omega_{0}\right)=W^{A C}$.

The Laplacian $\mathbb{L}(s)=B \cdot W(s) \cdot B^{T}$, partitioned as in (1), is

$$
\mathbb{L}(s)=\left(\begin{array}{ll}
\mathbb{L}_{b b}(s) & \mathbb{L}_{b \ell}(s) \\
\mathbb{L}_{\ell b}(s) & \mathbb{L}_{\ell \ell}(s)
\end{array}\right)
$$

with

$$
\begin{aligned}
& \mathbb{L}_{b b}(s)=\left(\begin{array}{ccc}
\frac{10}{1+0.02 s} & 0 & 0 \\
0 & \frac{5}{1+0.035 s} & 0 \\
0 & 0 & \frac{1}{1+0.01 s}
\end{array}\right), \\
& \mathbb{L}_{b \ell}(s)=\left(\begin{array}{c}
-\frac{10}{1+0.02 s} \\
-\frac{5}{1+0.035 s} \\
-\frac{1}{1+0.01 s}
\end{array}\right), \quad \mathbb{L}_{\ell b}(s)=\left(\mathbb{L}_{b \ell}(s)\right)^{T},
\end{aligned}
$$

and

$$
\mathbb{L}_{\ell \ell}(s)=\frac{16(1+0.011 s)(1+0.030 s)}{(1+0.01 s)(1+0.02 s)(1+0.035 s)} .
$$

The accompanying matrix $\mathcal{T}(s)$ is obtained from (9b) as

$$
\mathcal{T}(s)=\left(\begin{array}{c}
\frac{0.625(1+0.01 s)(1+0.035 s)}{(1+0.030 s)(1+0.011 s)} \\
\frac{0.312(1+0.01 s)(1+0.02 s)}{(1+0.030 s)(1+0.011 s)} \\
\frac{0.063(1+0.02 s)(1+0.035 s)}{(1+0.011 s)(1+0.030 s)}
\end{array}\right) .
$$

We can now characterize $\mathcal{G}_{\text {red }}^{\mathcal{A}}$ computed with respect to $\omega_{0}$, and then compute $\mathbb{L}_{\text {red }}^{\mathcal{A}}(s)$. Sets $\mathcal{V}_{b}$ and $\mathcal{E}_{\text {red }}$ are the same as in Example 1, whereas, following Definition 5, the weights matrix $W_{\text {red }}^{\mathcal{A}}(s)$ is obtained applying (11) to the Laplacian $\mathbb{L}_{\text {red }}^{A C}$ in (7). Consequently, one has

$$
\begin{aligned}
W_{\text {red }}^{\mathcal{A}}(s) & =\operatorname{diag}\left(W_{\text {red }, 12}^{\mathcal{A}}(s), W_{\text {red }, 13}^{\mathcal{A}}(s), W_{\text {red }, 23}^{\mathcal{A}}(s)\right)= \\
& =\left(\begin{array}{ccc}
\frac{3.642}{1+0.038 s} & 0 & 0 \\
0 & \frac{0.69}{1+0.010 s} & 0 \\
0 & 0 & \frac{0.254}{1+0.013 s}
\end{array}\right)
\end{aligned}
$$

where $W_{\text {red,12 }}^{\mathcal{A}}(s), W_{\text {red,13 }}^{\mathcal{A}}(s), W_{\text {red,23 }}^{\mathcal{A}}(s)$ are the admittance transfer functions of the reduced edges $r_{1}, r_{2}, r_{3}$, respectively (see Figure 2b). Next, let

$$
B_{\text {red }}=\left(\begin{array}{ccc}
-1 & -1 & 0 \\
1 & 0 & -1 \\
0 & 1 & 1
\end{array}\right)
$$

be the incidence matrix of the reduced network in Figure $2 b$. The Laplacian $\mathbb{L}_{\text {red }}^{\mathcal{A}}(s)$ of $\mathcal{G}_{\text {red }}^{\mathcal{A}}$ is given by

$$
\begin{aligned}
\mathbb{L}_{\text {red }}^{\mathcal{A}}(s)= & B_{\text {red }} \cdot W_{\text {red }}^{\mathcal{A}}(s) \cdot B_{\text {red }}^{T}= \\
= & \left(\begin{array}{lll}
\mathbb{L}_{\text {red,11 }}^{\mathcal{A}}(s) & \mathbb{L}_{\text {red,12 }}^{\mathcal{A}}(s) & \mathbb{L}_{\text {red,13 }}^{\mathcal{A}}(s) \\
\mathbb{L}_{\text {red,12 }}^{\mathcal{A}}(s) & \mathbb{L}_{\text {red,22 }}^{\mathcal{A}}(s) & \mathbb{L}_{\text {red,23 }}^{\mathcal{A},}(s) \\
\mathbb{L}_{\text {red,13 }}^{\mathcal{A}}(s) & \mathbb{L}_{\text {red,23 }}^{\mathcal{A}}(s) & \mathbb{L}_{\text {red,33 }}^{\mathcal{A},}(s)
\end{array}\right),
\end{aligned}
$$

with

$$
\begin{aligned}
& \mathbb{L}_{\text {red,11 }}^{\mathcal{A}}(s)=\frac{4.332(1+0.015 s)}{(1+0.038 s)(1+0.010 s)} \\
& \mathbb{L}_{\text {red,12 }}^{\mathcal{A}}(s)=-\frac{3.642}{(1+0.038 s)} \\
& \mathbb{L}_{\text {red,13}}^{\mathcal{A}}(s)=-\frac{0.690}{(1+0.010 s)} \\
& \mathbb{L}_{r e d, 22}^{\mathcal{A}}(s)=\frac{3.896(1+0.015 s)}{(1+0.038 s)(1+0.013 s)} \\
& \mathbb{L}_{r e d, 23}^{\mathcal{A}}(s)=-\frac{0.254}{(1+0.013 s)} \\
& \mathbb{L}_{r e d, 33}^{\mathcal{A}}(s)=\frac{0.945(1+0.012 s)}{(1+0.010 s)(1+0.013 s)}
\end{aligned}
$$

Finally, equivalent boundary currents are obtained substituting (15) in (12a) and (8) in (12b) (aAC-KR case), or replacing (15) in (13) and (14) in (10) (hKR case).

C. Asymptotic equivalence between original and reduced network models

The next Proposition characterizes the asymptotic behaviors preserved by aAC-KR and hKR.

Proposition 1. Consider the network represented by the graph $\mathcal{G}$, whose Laplacian is partitioned as in (1), and assume parameters $\tilde{R}_{i j}$ and $\tilde{L}_{i j}$ obtained through (11) with respect to $\omega_{0}$ are strictly positive. Then,

(i) if $I_{\ell}$ and $V_{b}$ are in PSSS with angular frequency $\omega_{0}$, then the asymptotic behavior of $I_{b}$ computed from (1) is the same as when $I_{b}$ is computed through aAC-KR (i.e. using (12)); 
(ii) if $V_{b}$ is in PSSS with angular frequency $\omega_{0}$, then the asymptotic behavior of $I_{b}$ computed from (1) is the same as when $I_{b}$ is computed through hKR (i.e. using (13)).

Proof of (i). From the strict positivity of $R_{i j}$ and $L_{i j}$ in (2), all entries $W_{i j}(s)$ of $L(s)$ have asymptotically stable poles. Hence, $L(s)$ is asymptotically stable. Since $I_{\ell}$ and $V_{b}$ are sinusoidal with angular frequency $\omega_{0}$, by virtue of the frequency response theorem [27], also the currents $I_{b}$ in (1) will reach a PSSS. In particular, it holds $\bar{J}_{\ell}(t)=\mathscr{A}\left(I_{\ell}(t)\right)=\vec{I}_{\ell}$, by definition of analytic signal. Therefore, if we write (12a) in time domain at frequency $\omega_{0}$, we get that the relation between phasors $\vec{V}_{b}, \vec{I}_{b}$ and $\vec{I}_{\ell}$ is

$$
\overrightarrow{I_{b}}=\underbrace{\mathbb{L}_{\text {red }}^{\mathcal{A}}\left(\mathrm{i} \omega_{0}\right)}_{\mathbb{L}_{\text {red }}^{A C}} \vec{V}_{b}-\mathcal{T}^{A C} \vec{I}_{\ell}
$$

that is (4b). Concluding, we have retrieved the AC-KR case, where the equivalence between original and reduced models is always guaranteed [4].

Proof of (ii). After applying hKR, the Laplace transforms of signals $I_{b}(t), V_{b}(t)$ and $I_{\ell}(t)$ are related by

$$
I_{b}(s)=\mathbb{L}_{\text {red }}^{\mathcal{A}}(s) V_{b}(s)-\mathcal{T}(s) I_{\ell}(s),
$$

where $\mathcal{T}(s)$ is given by (9b), and $\mathbb{L}_{\text {red }}^{\mathcal{A}}$ is the Laplacian of approximate Kron reduced graph $\mathcal{G}_{\text {red }}^{\mathcal{A}}$ with weights $W_{\text {red,ij }}(s)$ (see Definition 5). We note that, by hypothesis, the poles of all entries of $\mathbb{L}_{\text {red }}^{\mathcal{A}}$ have strictly negative real parts. Let us denote $I_{b}(s)$ and $\hat{I}_{b}(s)$ as the boundary currents computed from (1) and (13), respectively, and let us introduce $I_{\Delta}(s)=$ $I_{b}(s)-\hat{I}_{b}(s)$. Then, by (1) and (13), one has:

$$
I_{\Delta}(s)=\left(\mathbb{L}_{\text {red }}(s)-\mathbb{L}_{\text {red }}^{\mathcal{A}}(s)\right) V_{b}(s) .
$$

Suppose the inputs $V_{b}$ are sinusoids with angular frequency $\omega_{0}$. By the Frequency Response Theorem [27], each element of $\mathbb{L}_{r e d}(s) V_{b}(s)$ and $\mathbb{L}_{r e d}^{\mathcal{A}}(s) V_{b}(s)$ tends to a sinusoid, so that the relation between the phasors $\vec{I}_{\Delta}$ and $\vec{V}_{b}$ is given by $\vec{I}_{\Delta}=\left(\mathbb{L}_{r e d}\left(\mathrm{i} \omega_{0}\right)-\mathbb{L}_{\text {red }}^{\mathcal{A}}\left(\mathrm{i} \omega_{0}\right)\right) \vec{V}_{b}$. However, $\mathbb{L}_{\text {red }}\left(\mathrm{i} \omega_{0}\right)=$ $\mathbb{L}_{\text {red }}^{\mathcal{A}}\left(\mathrm{i} \omega_{0}\right)$ by construction, hence $\vec{I}_{\Delta}=0$, which means that $I_{\Delta} \rightarrow 0$ asymptotically.

\section{Generalization to three-phase linear networks in dq coor- dinates}

All the considerations reported in Sections II and III so far (along with the related formulae) hold, without restrictions, for any linear network. In this paragraph, we focus on threephase linear networks in $d q$ coordinates. From this point on, we assume balanced $R L$ lines, and three-phase electrical signals without zero-sequence components. It follows that, under these circumstances, we can split the circuit into three independent and identical single-phase circuits. Each one is associated with the equivalent "single-phase" directed graph $\mathcal{G}^{s p}=\left(\mathcal{V}^{s p}, \mathcal{E}^{s p}, W^{s p}(s)\right)$ where transfer functions $W_{i j}^{s p}(s)=1 /\left(R_{i j}+s L_{i j}\right)$ represent, independently of the phase $\star \in\{a, b, c\}$, the relation between $\mathscr{L}\left[V_{i}^{\star}(t)-V_{j}^{\star}(t)\right]$ and $\mathscr{L}\left[I_{i j}^{\star}(t)\right],(i, j) \in \mathcal{E}^{s p}$.

Three-phase signals can be conveniently represented in the $d q 0$ coordinates. In this case, under our assumptions, the graph
$\mathcal{G}$ representing the considered three-phase network has the same topology of the corresponding single-phase circuit $\mathcal{G}^{s p}$. Moreover, it can be shown that, in the $d q 0$ reference frame, the $R L$ line associated with the edge $(i, j) \in \mathcal{E}^{s p}$ has the dynamics

$$
\frac{\mathrm{d}}{\mathrm{d} t} I_{i j}^{d q}=-\left(\frac{R_{i j}}{L_{i j}}+\mathrm{i} \omega_{0}\right) I_{i j}^{d q}+\frac{1}{L_{i j}}\left(V_{i}^{d q}-V_{j}^{d q}\right)
$$

and its corresponding transfer function has the form $I_{i j}^{d q}(s)=$ $W_{i j}(s)\left(V_{i}^{d q}(s)-V_{j}^{d q}(s)\right)$ with

$$
W_{i j}(s)=\frac{1}{Z_{i j}+s L_{i j}}, \quad Z_{i j}=R_{i j}+\mathrm{i} \omega_{0} L_{i j} .
$$

Also in this case, the current-balance equations $I^{d q}(s)=$ $\mathbb{L}^{d q}(s) V^{d q}(s)$ can be partitioned as in (1), with $I^{d q}=$ $\left[I_{b}^{d q^{T}}, I_{\ell}^{d q^{T}}\right]^{T}$ and $V^{d q}=\left[V_{b}^{d q^{T}}, V_{\ell}^{d q^{T}}\right]^{T}$. In $d q$ coordinates, instantaneous $\mathrm{KR}$ is characterized analogously to Section II-B. Moreover, also aAC-KR and hKR can be redefined in $d q$ coordinates; notably, by performing $\mathrm{AC}-\mathrm{KR}$ on $\mathcal{G}^{s p}$, one obtains the topology of the approximate Kron reduced graph $\mathcal{G}_{\text {red }}^{\mathcal{A}}$ in $d q$ reference frame, as well as the reduced lines parameters $\tilde{R}_{i j}$ and $\tilde{L}_{i j}$ characterizing its weights

$$
\begin{gathered}
W_{r e d, i j}^{\mathcal{A}}(s)=\frac{1}{\tilde{Z}_{i j}+s \tilde{L}_{i j}}, \\
\tilde{Z}_{i j}=-\left(\mathbb{L}_{r e d, i j}^{A C}\right)^{-1}=\tilde{R}_{i j}+\mathrm{i} \omega_{0} \tilde{L}_{i j}, \quad \tilde{L}_{i j}=\frac{1}{\omega_{0}} \operatorname{Im}\left(\tilde{Z}_{i j}\right) .
\end{gathered}
$$

As regards the computation of the equivalent internal currents to be injected into the boundary nodes of the reduced network, (12b) and (10) become, respectively

$$
\tilde{J}_{b}^{d q}(s)=\mathscr{L}\left[T(\theta) \cdot \operatorname{Re}\left(\mathcal{T}^{A C} \bar{J}_{\ell}^{a b c}(t)\right)\right]
$$

and

$$
\tilde{I}_{b}^{d q}(s)=\mathcal{T}(s) I_{\ell}^{d q}(s),
$$

depending on whether we are using aAC-KR or hKR.

\section{NUMERICAL EXAMPLES}

In this Section, we assess the features of aAC-KR and hKR through numerical examples. In particular, we consider the three-phase network in Figure 3a, composed of three ideal voltage sources and balanced $R L$ lines connecting the generators to a common load. In this simple case, both aAC$\mathrm{KR}$ and $\mathrm{hKR}$ amount to a $\mathrm{Y}-\Delta$ transformation. The reduced network is shown in Figure 3b. Since the corresponding threephase balanced voltage generators are identical for the original and reduced networks, from Proposition 1 we expect $I_{b}$ in the original and reduced models to be the same, if a PSSS is reached. The lines and loads parameters, as well as the voltages of generators, are collected in [31]. 


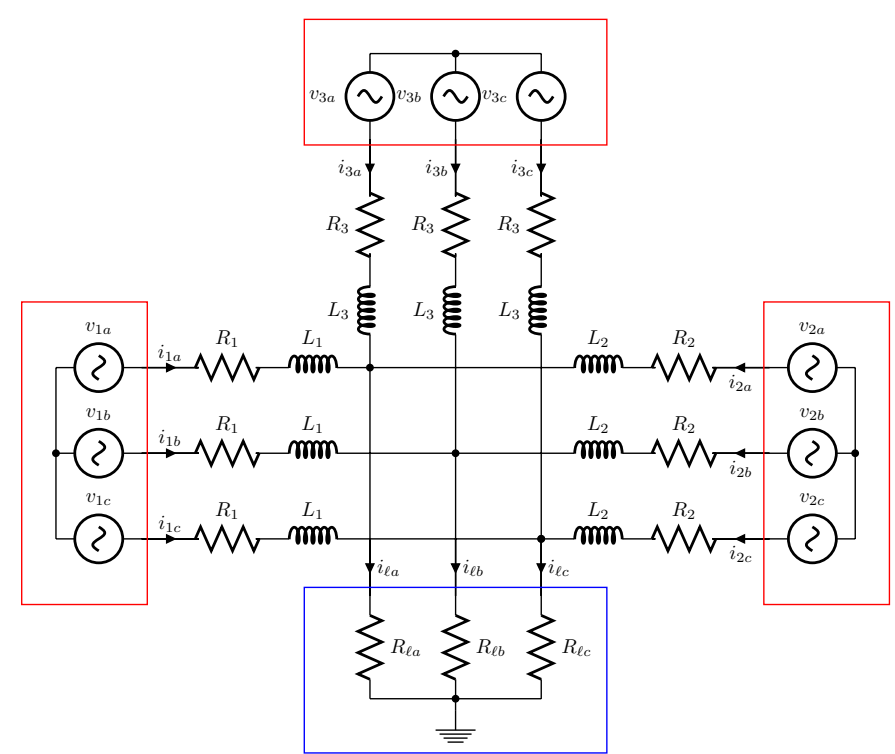

(a) Original network

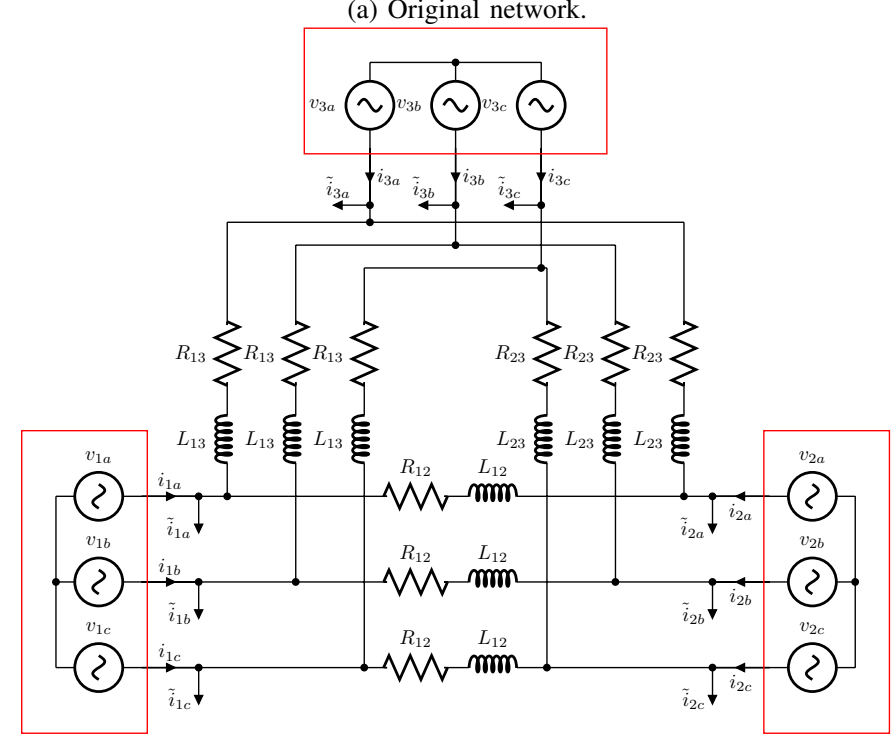

(b) Reduced model obtained via approximate KR methods.

Fig. 3: Numerical examples: original and reduced networks. Red and blue boxes enclose boundary and interior nodes, respectively.

1) Example 1 - Linear unbalanced load: In this example, we assume that the resistive load is unbalanced. Figures $4 a-4 b$ compare the original output current of phase $a$ of generator 1 with its corresponding signal in the reduced networks obtained through aAC-KR and hKR, respectively. The plots reveal that the proposed methods ensure asymptotic equivalence, even if the load is unbalanced but a PSSS is achieved. In particular, from Figures $4 \mathrm{c}-4 \mathrm{~d}$, we notice that both the aAC-KR and hKR model errors go to zero after short transients. Analogous results are obtained for all the other phases and nodes.

2) Example 2 - Nonlinear load: For this second example, we replace the resistive load in Figure 3a by a six-pulse bridge rectifier. As in the previous example, we compare the currents at boundary nodes of the original circuit with those of the reduced models and, for the sake of simplicity, we concentrate on the output currents of generator 1, phase $a$ (see Figures $5 a-5 b)$. In particular, from Figure $5 c$, one can see that the aAC-KR model error increases substantially with respect to Example 1, while the one of the hybrid Kron reduced network goes asymptotically to zero (see Figure 5d). We highlight that latter phenomenon is predicted by point (ii) of Proposition 1.

Analogous results are obtained for all the other phases and generators.

Remark 3. The proposed approximate methods produce similar results in networks reaching PSSS regime at frequency $\omega_{0}$, but $h K R$ provides better performances (i.e. faster convergence and lower errors) than $a A C-K R$ in case of networks with nonlinear loads. This is due to the fact that $\tilde{I}_{b}^{d q}$ in (13) represents the "true" internal currents mapped to boundary nodes (they are the same as in equation (10)). Indeed, $h K R$ differs from KR (9) only in the dynamics of the branches, and not in the equivalent internal currents. On the other hand, as highlighted in Section III-B, this better accuracy of $h K R$ with respect to aAC-KR comes at the expenses of an higher implementation complexity.

\section{KRON REDUCTION OF AC IMGS}

In this Section, we show how to apply the approximate KR methods described in Section III to the control of AC Islanded microGrids (ImGs), i.e. a group of interconnected Distributed Generation Units (DGUs) and loads. Our aim is to exploit aAC-KR and hKR to generalize the Plug-and-Play $(\mathrm{PnP})$ algorithm described in [20] for designing decentralized controllers for AC ImGs guaranteeing voltage and frequency stability. The method in [20], in fact, assumes (i) loadconnected ImG topologies (i.e. with loads appearing only at the output terminals of each inverter), and (ii) $R L$ power lines connecting DGUs. In this context, we can resort to one of our approximate KR method to map arbitrarily interconnected ImGs into their corresponding load-connected networks (by labeling as internal nodes those representing loads that are not directly attached to DGUs). Then, since both aAC-KR and hKR preserve the $R L$ structure of the reduced lines, we can use the procedure in 11 to design PnP regulators with respect to the equivalent simplified circuit.

In the sequel, we first introduce the graph associated with an ImG, together with the electrical models of DGUs and lines used for controller synthesis. Then, the PnP algorithm in [20] is briefly summarized before describing how to combine this design procedure with the proposed approximate KR methods.

\section{A. ImG associated graph}

As for any linear electrical network, we can associate to an $\operatorname{ImG}$ a weighted directed graph $\mathcal{G}=(\mathcal{V}, \mathcal{E}, W(s))$ (see the example in Figure 6) where the vertex set $\mathcal{V}$ is partitioned into boundary and internal nodes. In this case, the subset $\mathcal{V}_{b}$ identifies Point of Common Coupling (PCC) nodes, i.e. the output terminals of each DGU, while $\mathcal{V}_{\ell}$ contains load nodes. Nodal currents injected by the DGUs and loads are denoted with $I_{b}$ and $I_{\ell}$, respectively, and they are positive if entering into the nodes. Nodal voltages are denoted analogously. As 


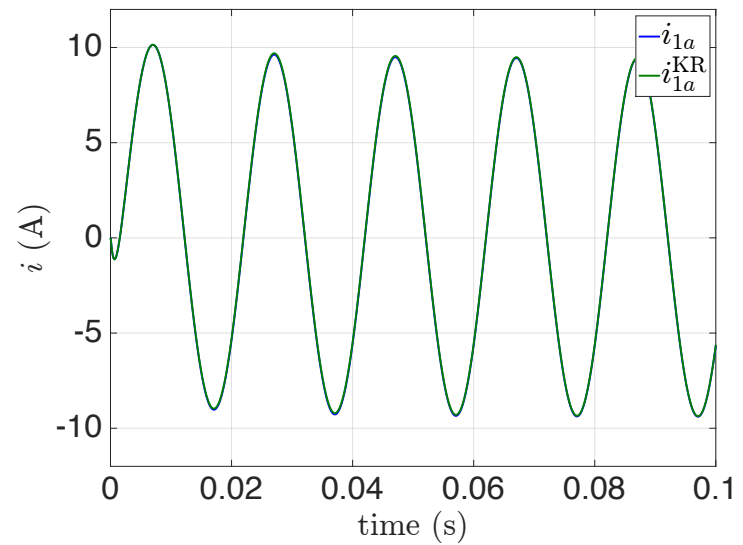

(a) Comparison between original and reduced current of phase $a$ currents of generator 1 - aAC-KR.

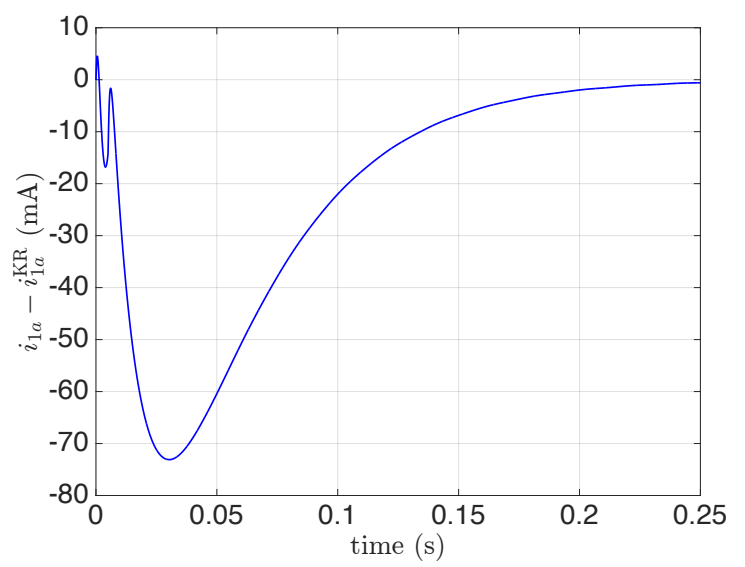

(c) Error between original and reduced current of phase $a$ currents of generator 1 - aAC-KR.

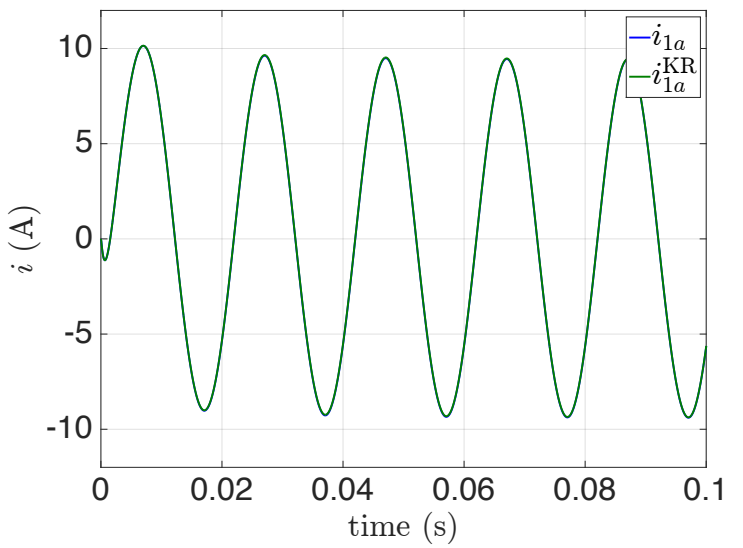

(b) Comparison between original and reduced current of phase $a$ currents of generator 1 - hKR.

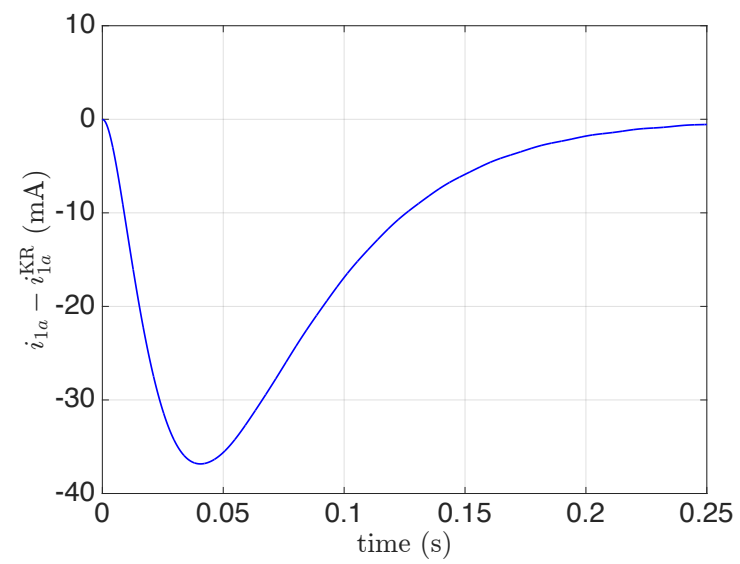

(d) Error between original and reduced current of phase a currents of generator 1 - hKR

Fig. 4: Example 1 - Evaluation of the output currents generated through aAC-KR and hKR, in presence of a linear load.

regards the edges, they correspond to $R L$ power lines with arbitrary orientation and same sign convention as in Section II.

\section{B. DGU and line electrical models}

We assume three-phase electrical signals without zero sequence components and balanced network parameters ${ }^{2}$. Note that we do not assume balanced signals; hence, the case of unbalanced load currents is included in this framework.

Let $\omega_{0}$ be the reference network frequency. The model of DGU $i \in \mathcal{V}_{b}$ in $d q$ coordinates is [33]:

$$
\left\{\begin{aligned}
\frac{\mathrm{d}}{\mathrm{d} t} V_{i}^{d q} & =-\mathrm{i} \omega_{0} V_{i}^{d q}+\frac{I_{t i}^{d q}}{C_{t i}}-\frac{I_{L i}^{d q}}{C_{t i}}-\frac{1}{C_{t i}} I_{b i}^{d q} \\
\frac{\mathrm{d}}{\mathrm{d} t} I_{t i}^{d q} & =-\left(\frac{R_{t i}}{L_{t i}}+\mathrm{i} \omega_{0}\right) I_{t i}^{d q}-\frac{V_{i}^{d q}}{L_{t i}}+\frac{V_{t i}^{d q}}{L_{t i}}
\end{aligned}\right.
$$

where $V_{i}, I_{t i}, I_{b i}, I_{L i}, V_{t i}, R_{t i}, C_{t i}$ and $L_{t i}$ are shown in the equivalent single-phase electrical scheme in Figure 7.

${ }^{2}$ See, e.g., [32] for basic definitions.
Remark 4. The scheme in Figure 7 reveals that each DGU can have a load current $I_{L i}$ connected to its PCC. These local loads, which will be treated as exogenous disturbances in control design, are different from load currents $I_{\ell}$ defined in Section V-A. The latter ones, in fact, identify loads that are not directly connected to PCCs (see, e.g., the blue circles in Figure 6).

Consider an ImG composed of $n$ nodes partitioned into $n_{b}$ boundary nodes and $n_{\ell}$ internal nodes. If $n=n_{b}$, then $n_{\ell}=$ 0 , which means that we retrieve a load-connected $\operatorname{ImG}$, with load currents appearing only at the PCCs. Otherwise, we can write nodal currents and voltages as $I^{d q}=\left[I_{b}^{d q^{T}}, I_{\ell}^{d q^{T}}\right]^{T}$ and $V^{d q}=\left[V_{b}^{d q^{T}}, V_{\ell}^{d q^{T}}\right]^{T}$, respectively. At this point, as already shown, it is possible to express the current-balance equations as $I^{d q}(s)=\mathbb{L}(s) V^{d q}(s)$, where $\mathbb{L}(s)$ is the Laplacian of the graph $\mathcal{G}$ associated with the $\operatorname{ImG}$, with weights $W_{i j}(s)$ in the form (17). 


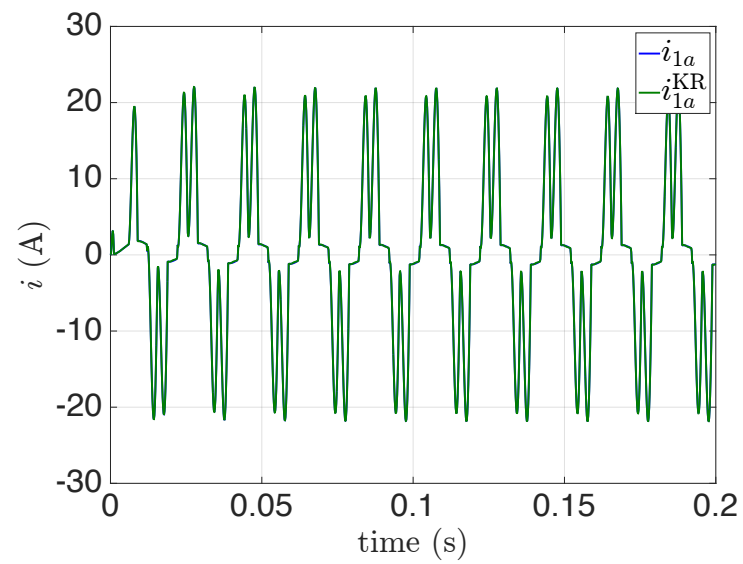

(a) Original (in blue) and reduced (in green) currents of phase $a$ of generator 1 - aAC-KR

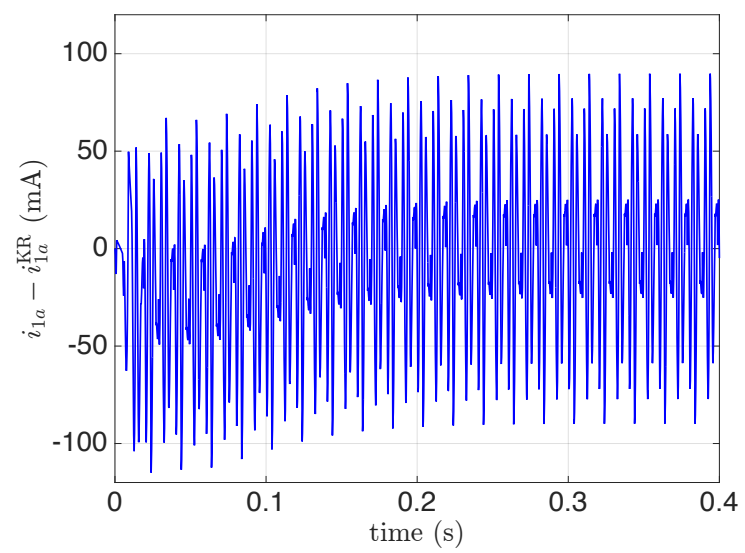

(c) Error between original and reduced current of phase $a$ currents of generator 1 - aAC-KR.

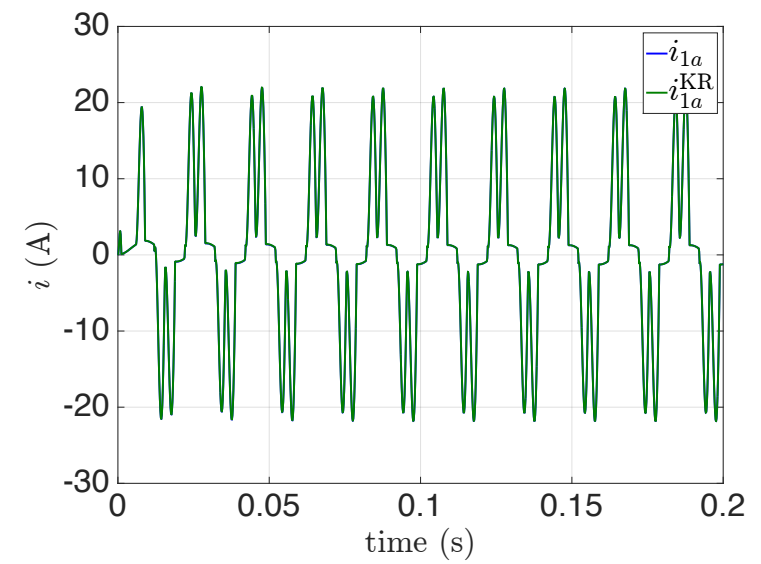

(b) Original (in blue) and reduced (in green) currents of phase $a$ of generator 1 - hKR.

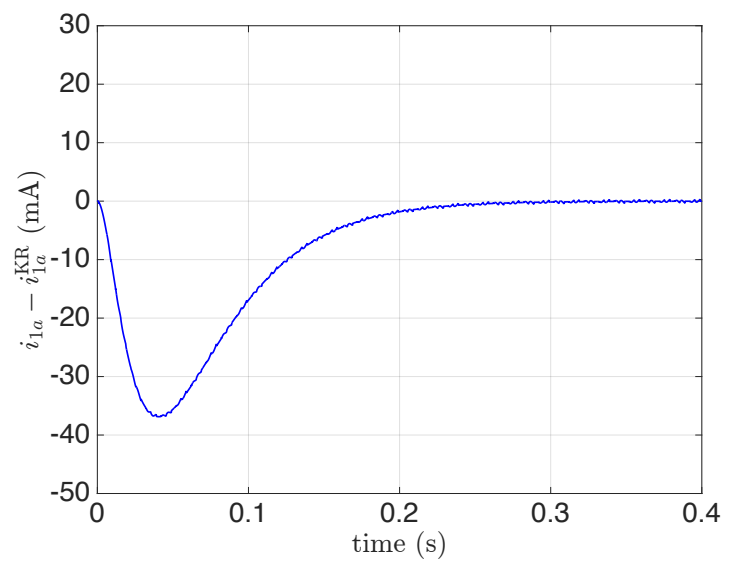

(d) Error between original and reduced current of phase a currents of generator $1-\mathrm{hKR}$.

Fig. 5: Example 2 - Evaluation of the features of aAC-KR and hKR in presence of a nonlinear load.

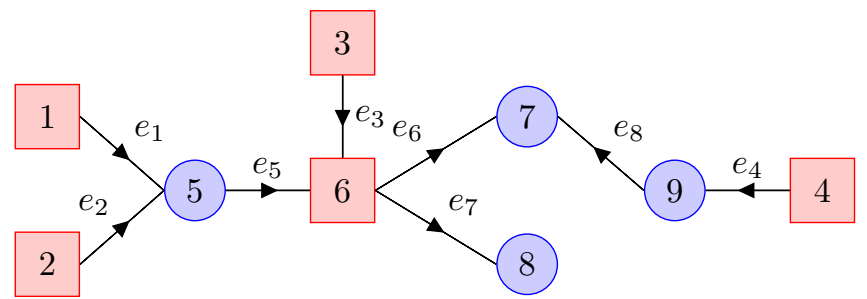

Fig. 6: Example of a graph associated with an ImG. Red squares denote DGUs (i.e. boundary nodes), while blue circles represent loads (i.e. internal nodes).

\section{PnP design for load-connected ImGs}

In the following, we summarize the PnP algorithm in [20] for designing decentralized controllers guaranteeing voltage and frequency stability in an ImG. Local regulators $\mathcal{C}_{i}$ use measurements of the voltage $V_{i}^{d q}$ at PCC (see Figure 7) and the current $I_{t i}^{d q}$ to control the voltage $V_{t i}^{d q}$ at the $i$-th Voltage Source Converter (VSC) so as to make $V_{i}^{d q}$ track a reference signal. Furthermore, each controller is composed of a matrix gain and an integral action on the $d$ and $q$ components of the

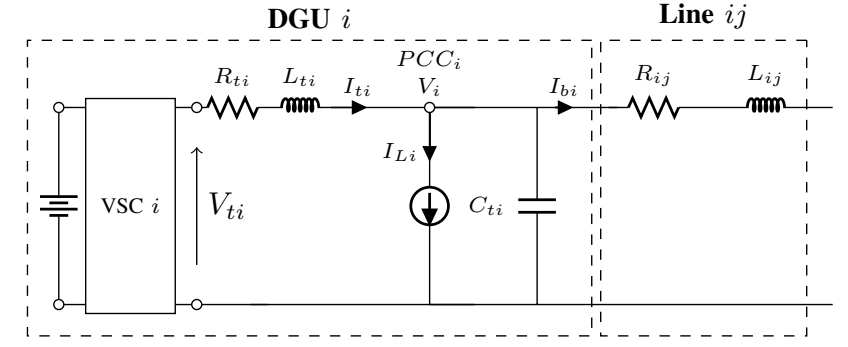

Fig. 7: Single-phase equivalent circuit of DGU $i$ composed of a Voltage Source Converter (VSC), an $R L C$ filter, a local load $I_{L i}$ and a single $R L$ line connecting DGU $i$ with the node $j \in \mathcal{V}$.

tracking error.

When a DGU (say DGU $i$ ) wants to join the network (e.g. DGU 3 in Figure 8), it issues a plug-in request to its future neighbors, i.e. DGUs $j \in \mathcal{N}_{i}$ (see, for example, DGUs 2 and 4 in Figure 8). DGU $i$ then solves the Linear Matrix Inequality (LMI) problem (19) in [20], which depends 


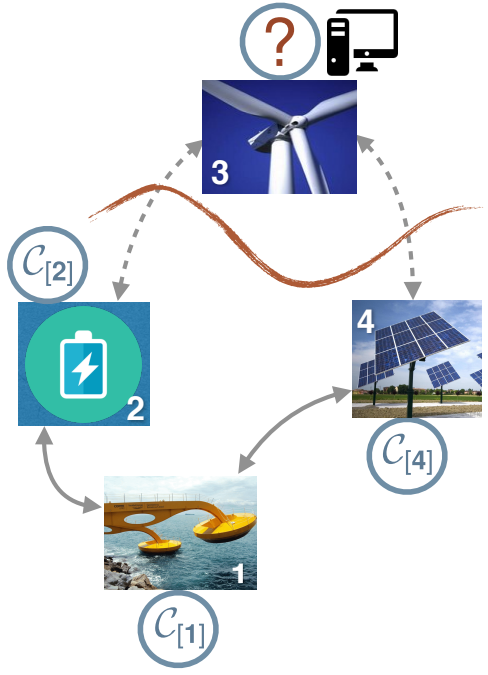

Fig. 8: Example of plug-in request issued by DGU 3 to its future neighbors (i.e. DGUs 2 and 4).

only upon the parameters of the lines $i j^{3}$. If feasible, the optimization problem produces a controller $\mathcal{C}_{i}$, along with a local and structured Lyapunov function that can be used for certifying stability of the whole ImG. Since also DGUs $j \in \mathcal{N}_{i}$ will have a new neighbor, they must update their controller $\mathcal{C}_{j}$ by tacking into account the parameters of the new line $j i$. This is done by solving an LMI problem analogous to the one solved by DGU $i$. If one of the above LMI problems is unfeasible, plug-in of DGU $i$ is denied. Otherwise, as shown in [20, Proposition 3], DGU $i$ can be connected and stability of the whole ImG can be certified using the sum of the computed local Lyapunov functions.

Unplugging of a DGU (say DGU $m$ ) follows a similar procedure: as line $m k, k \in \mathcal{N}_{m}$ will be disconnected from the corresponding DGU $k$, all controllers $\mathcal{C}_{k}$ must be successfully redesigned before allowing the disconnection. Following a similar reasoning, also the possibility of changing a parameter of line $i j$ (or add a new line $i j$ ), must be first tested by successfully designing controllers $\mathcal{C}_{i}$ and $\mathcal{C}_{j}$ through suitable LMIs.

Remark 5. While line changes are not common in ImGs, they could happen in the equivalent reduced network because of the addition/removal of load nodes in the original ImG. Examples of this phenomenon are provided in the next Section.

\section{PnP design for ImGs with general topologies}

Now we show how to extend the design algorithm in [20] to ImGs with arbitrary topologies. Let us $\mathcal{G}$ be the directed graph associated with an ImG and let us assume we have selected one KR method among aAC-KR and hKR. When the plug-in of a DGU or a load node (say node $i$ ) is required, one should first update $\mathcal{G}$ accordingly, thus obtaining the graph $\mathcal{G}^{\text {new }}$. Then, the chosen approximate method is applied to $\mathcal{G}^{\text {new }}$ for obtaining

\footnotetext{
${ }^{3}$ Each LMI problem involves only 25 scalar variables and it can be easily solved with state-of-art packages for convex optimization.
}

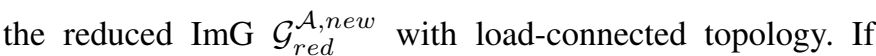
some resistances or inductances of reduced lines are negative, the plugging-in of node $i$ is denied, as one of the assumption of the PnP algorithm in [20] is not fulfilled. Otherwise, one compares the reduced graphs $\mathcal{G}_{\text {red }}^{\mathcal{A}}$ (associated with $\mathcal{G}$ ) and $\mathcal{G}_{\text {red }}^{\mathcal{A}, \text { new }}$ for finding the set $\mathcal{U} \subseteq \mathcal{V}_{b}$ of DGUs that have new neighbors or that are connected to lines whose impedance has changed. The LMI problem (19) in [20] is then solved for all DGUs $j \in \mathcal{U}$ (and also for $j=i$, if node $i$ is a DGU), hence producing new controllers $\mathcal{C}_{j}$. If no LMI is infeasible, controllers in the original ImG are updated and connection of node $i$ is allowed.

Unplugging of a node can be performed in a similar way.

Remark 6. According to the above algorithm, $a A C-K R$ and $h K R$ are performed in a centralized fashion every time there is a change in the network topology. This is in contrast with PnP design, whose main feature is to avoid any centralized computations. In the future, we will study how to perform the proposed approximate KR methods in a distributed fashion. Notably, one can develop this generalization according to the iterative KR procedure [3] proposed for both AC networks in PSSS and resistive networks. In a similar spirit, we will study how to avoid the centralized computation of the set $\mathcal{U}$ by exploiting existing distributed algorithms for path-finding over directed graphs.

\section{Simulation OF A 21-BUS NETWORK}

In this Section, we assess the capability of PnP control and approximate KR methods to deal with networks characterized by complex topologies. Since we are interested in evaluating only the stabilizing effect of PnP controllers, local pre-filters and compensators have not been implemented. Now, recalling that (i) each compensator takes as input the load current $I_{L i}$ absorbed at the corresponding PCC (see [20] for details), and (ii) the proposed approximate $\mathrm{KR}$ methods differ only in the way equivalent internal currents referred to PCCs are computed, in this scenario it is equivalent to use aAC-KR or $\mathrm{hKR}$, as they both provide the same reduced graph $\mathcal{G}_{\text {red }}^{\mathcal{A}}$.

We use a network derived from the top half of IEEE 37 topology [21] and identify generation nodes and loads as in [34]. The simulation has been performed in PSCAD.

\section{A. ImG topology}

The ImG in Figure 9 has 21 nodes, with six DGUs, electrical $R L$ lines having time constants spread in a wide range, linear $R$ and $R L$ loads, as well as highly nonlinear and highly inductive loads.

Compared to the IEEE 37 network, a switch $S W_{1}$ has been introduced, allowing the plugging-in/unplugging of loads at nodes 16, 17 and 18. Moreover, two branches $\left(e_{18}\right.$ and $\left.e_{19}\right)$ were added and connected to the microgrid through switches $S W_{2}$ e $S W_{3}$, respectively. The edge $e_{18}$ creates a mesh between DGUs 1, 3 and 4; this allows us to show that PnP controllers can also stabilize meshed networks. The edge $e_{19}$ simply changes the impedance between DGUs 3 and 5 (as long as $S W_{1}$ is closed). Finally, one generation node (vertex 


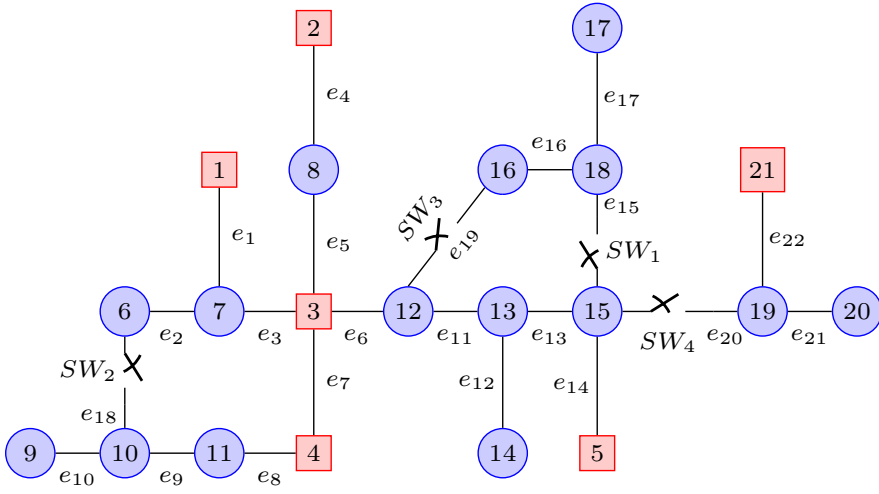

Fig. 9: 21-bus network: red squares denote boundary nodes (DGUs), blue circles represent internal nodes (loads).

21) and two loads (at nodes 19 and 20) have been introduced, so as to simulate the plugging-in of a new DGU. The new generation unit is connected to the ImG via switch $S W_{4}$.

At time $t=0 \mathrm{~s}$, there is no energy stored in all the inductors and capacitors and all the switches are open. At $t=5 \mathrm{~s}$, switch $S W_{1}$ closes, causing an increase in electrical loads, mainly supplied by DGUs 3 and 5. Next, $S W_{2}$ e $S W_{3}$ close at instants $t=6.5 \mathrm{~s}$ and $t=8 \mathrm{~s}$, respectively, connecting new branches to the network. Finally, at time $t=9.5 \mathrm{~s}$ switch $S W_{4}$ closes, so that the sixth generation unit is connected to the ImG. All the electrical parameters of the 21-bus network are collected in [31].

\section{B. PnP control design}

As described in Section V-D, the first step in control design consists in applying the chosen approximate method (aAC$\mathrm{KR}$ or hKR) to the original network, thus obtaining the equivalent load-connected model. In particular, the reduced line impedances are computed using (18b), with $\omega_{0}=2 \pi 50$ $\mathrm{rad} / \mathrm{s}$.

The topology of the reduced network actually depends on the state of the switches in the original network. As long as $S W_{2}, S W_{3}$ and $S W_{4}$ are open, the network in Figure 9 has a radial topology. Therefore, the equivalent impedance between nodes 3 and 5 is equal to the sum of the impedances of edges $e_{6}, e_{11}, e_{13}$ and $e_{14}$, irrespectively of the state of switch $S W_{1}$. On the contrary, when switches $S W_{2}, S W_{3}$ and $S W_{4}$ are closed, the topology of the Kron reduced network and its impedances change. Figure 10 collects the reduced networks that arise during the simulation. In particular, Figure 10a holds when all the four switches are open, or when only $S W_{1}$ is closed. The network in Figure 10b refers to the case when switches $S W_{2}$ and $S W_{3}$ become closed, while the diagram in Figure 10c holds when all the four switches are closed. We further highlight that the resistances and inductances of the Kron reduced circuits in Figure 10, collected in [31], are positive.

At time $t=5 \mathrm{~s}, S W_{1}$ closes, but the Kron reduced network does not change, and no redesign of the controllers is needed. In fact, the connection of load nodes 16,17 and 18 changes the term accounting for the effect of internal currents $\left(\tilde{J}_{b}(s)\right.$ in

aAC-KR or $\tilde{I}_{b}(s)$ in hKR), which is mapped into an additional contribution to the load at PCCs 3 and 5. This is, however, not critical since DGU loads are treated as disturbances by PnP controllers [20]. At instant $t=6.5 \mathrm{~s}$, the equivalent impedances between nodes 1,3 and 4 change; therefore, the controllers of DGUs 1, 3 and 4 must be redesigned (all the other controllers do not change). At instant $t=8 \mathrm{~s}$, the equivalent impedance between boundary nodes 3 and 5 changes: the corresponding DGUs must update their controllers. At $t=9.5$ s, DGU 6 is connected to the network: controllers of DGUs 3 e 5 must be redesigned again.

We highlight that the proposed controllers effectively stabilize voltage and frequency in the ImG. In particular, for plots of the frequency response and the eigenvalues of the closed loop ImG, we defer the reader to [31].

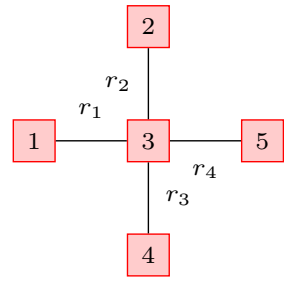

(a) Equivalent reduced network for $t<6.5$ s.

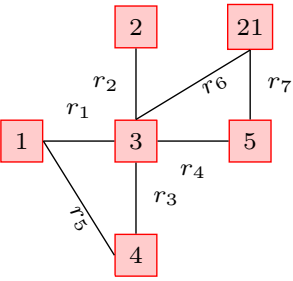

(c) Equivalent reduced network for $t \geq 9.5 \mathrm{~s}$.

Fig. 10: Simulation of a 21-bus ImG: Kron reduced networks.

\section{Simulation results}

The reference signals for all the generation units are $V_{d}^{\text {ref }}=$ $\sqrt{2} \cdot 230 \mathrm{~V}$ and $V_{q}^{r e f}=0 \mathrm{~V}$. Figure 11 shows the Root Mean Square (RMS) voltage, frequency and Total Harmonic Distortion $^{4}$ (THD) of phase $a$ at the PCCs of the boundary nodes (i.e nodes 1-5 and 21), respectively. We highlight that, in spite of all the variations of the ImG topology, PnP decentralized control ensures good tracking of voltage references for all DGUs (see Figure 11a). We note that real-time switch between different controllers has been implemented using a bumpless control transfer scheme similar to the one used in classical PID regulators [36]. This guarantees that (i) control variables do not have sudden variations at switching times and (ii) after transients due to switching, control variables are identical to those obtained without bumpless control transfer. In our case, bumpless controllers are effective in limiting voltage surges and dips to a few volts when updates of controllers take place (for $t \geq 4 \mathrm{~s}$, the maximal deviation from the reference RMS voltage is of less than 20 volts). Figure $11 \mathrm{~b}$ shows that the

\footnotetext{
${ }^{4}$ See [35] for a definition.
} 
impact of the topology commutations is minor also on the frequency profiles. In fact, PnP controllers promptly restore the frequencies to the nominal value, ensuring negligible variations (i.e. less than $0.5 \mathrm{~Hz}$ when the highly inductive load is connected and less than $0.1 \mathrm{~Hz}$ when other events occur). Finally, from Figure 11c, we notice that, apart from spikes due to the closing of switches, THD values are below the maximum limit (5\%) recommended in [35].

Overall, the fact that voltage and frequency stability is guaranteed even for such a complicated network, proves that the proposed approximate KR methods are well suited tools for extending the $\mathrm{PnP}$ scalable design to ImGs with arbitrary topologies.

\section{CONCLUSIONS}

In this paper, we introduced two approximate network reduction algorithms based on KR and capable to preserve exactly the asymptotic periodic behavior of voltages and currents at target nodes. We also used the proposed KR methods for extending the PnP control design presented in [20] to AC ImGs with arbitrary topology. As regards future developments, besides addressing the problems described in Remark 6, we aim to extend aAC-KR and hKR also to the case of DC ImGs equipped with PnP controllers [37]. Another interesting direction is the generalization of our results to the case of line models including capacitive elements.

\section{REFERENCES}

[1] G. Kron, Tensor analysis of networks. New York: John Wiley and Sons, 1939.

[2] A. Van der Schaft, "Characterization and partial synthesis of the behavior of resistive circuits at their terminals," Systems \& Control Letters, vol. 59, no. 7, pp. 423-428, 2010.

[3] F. Dörfler and F. Bullo, "Kron reduction of graphs with applications to electrical networks," IEEE Transactions on Circuits and Systems I: Regular Papers, vol. 60, no. 1, pp. 150-163, 2013.

[4] S. Y. Caliskan and P. Tabuada, "Kron reduction of power networks with lossy and dynamic transmission lines," in Proceedings of the 51st IEEE Annual Conference on Decision and Control (CDC), 2012. IEEE, 2012, pp. 5554-5559.

[5] - "Towards Kron reduction of generalized electrical networks," Automatica, vol. 50, no. 10, pp. 2586-2590, 2014.

[6] S. V. Dhople, B. B. Johnson, F. Dörfler, and A. O. Hamadeh, "Synchronization of nonlinear circuits in dynamic electrical networks with general topologies," IEEE Transactions on Circuits and Systems I: Regular Papers, vol. 61, no. 9, pp. 2677-2690, 2014.

[7] A. Morched, J. Ottevangers, and L. Marti, "Multi-port frequency dependent network equivalents for the emtp," IEEE Transactions on Power Delivery, vol. 8, no. 3, pp. 1402-1412, 1993.

[8] J. M. Guerrero, M. Chandorkar, T.-L. Lee, and P. C. Loh, "Advanced control architectures for intelligent microgrids, part I: decentralized and hierarchical control," IEEE Transactions on Industrial Electronics, vol. 60, no. 4, pp. 1254-1262, 2013.

[9] D. Kothari and I. Nagrath, Modern power system analysis. Tata McGraw-Hill Education, 2003.

[10] J. J. Grainger and W. D. Stevenson, Power system analysis, ser. McGrawHill Series in Electrical \& Computer Engineering. McGraw-Hill New York, 1994, vol. 31.

[11] J. Glover, M. Sarma, and T. Overbye, Power System Analysis \& Design. Cengage Learning, 2012.

[12] Y. Fan, G. Hu, and M. Egerstedt, "Distributed reactive power sharing control for microgrids with event-triggered communication," IEEE Transactions on Control Systems Technology, vol. 25, no. 1, pp. 118128, Jan 2017.

[13] A. Parisio, E. Rikos, and L. Glielmo, "A model predictive control approach to microgrid operation optimization," IEEE Transactions on Control Systems Technology, vol. 22, no. 5, pp. 1813-1827, Sept 2014.

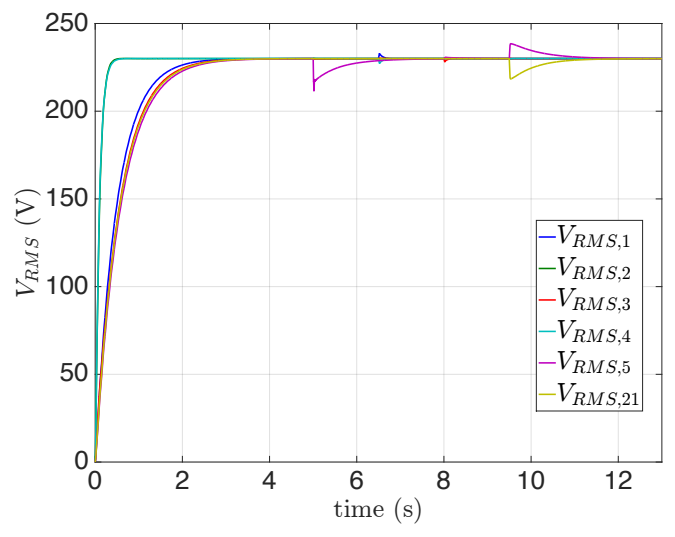

(a) RMS voltages at boundary nodes.

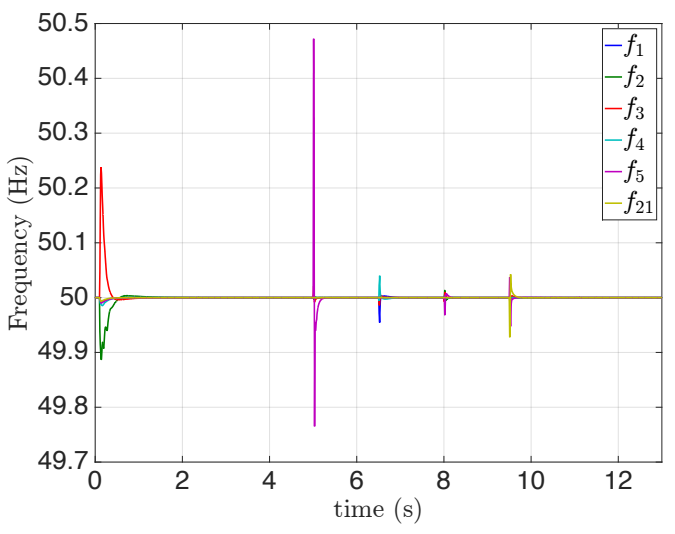

(b) Frequencies at boundary nodes.

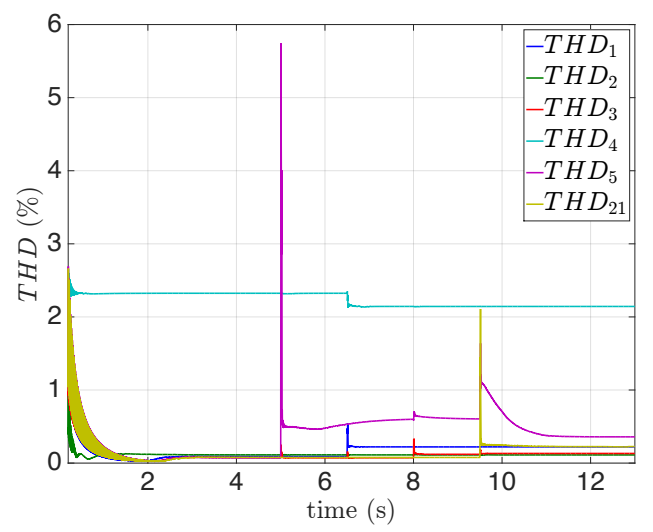

(c) THD at boundary nodes.

Fig. 11: Performance of $\mathrm{PnP}$ control and approximate $\mathrm{KR}$ methods with a 21-bus network. Switches $S W_{1}, S W_{2}, S W_{3}$ and $S W_{4}$ are closed at times $t=5 \mathrm{~s}, t=6.5 \mathrm{~s}, t=8 \mathrm{~s}$ and $t=9.5 \mathrm{~s}$, respectively.

[14] S. T. Cady, A. D. Domínguez-Garca, and C. N. Hadjicostis, "A distributed generation control architecture for islanded ac microgrids," IEEE Transactions on Control Systems Technology, vol. 23, no. 5, pp. 1717-1735, Sept 2015.

[15] S. R. Cominesi, M. Farina, L. Giulioni, B. Picasso, and R. Scattolini, "A two-layer stochastic model predictive control scheme for microgrids," IEEE Transactions on Control Systems Technology, to appear.

[16] C. D. Persis and N. Monshizadeh, "Bregman storage functions for microgrid control," IEEE Transactions on Automatic Control, to appear

[17] L. Luo and S. V. Dhople, "Spatiotemporal model reduction of inverter- 
based islanded microgrids," IEEE Transactions on Energy Conversion, vol. 29, no. 4, pp. 823-832, 2014.

[18] J. Schiffer, R. Ortega, A. Astolfi, J. Raisch, and T. Sezi, "Conditions for stability of droop-controlled inverter-based microgrids," Automatica, vol. 50, no. 10, pp. 2457-2469, 2014.

[19] J. M. Guerrero, J. Matas, L. García De Vicuña, M. Castilla, and J. Miret, "Decentralized control for parallel operation of distributed generation inverters using resistive output impedance," Industrial Electronics, IEEE Transactions on, vol. 54, no. 2, pp. 994-1004, 2007.

[20] S. Riverso, F. Sarzo, and G. Ferrari-Trecate, "Plug-and-play voltage and frequency control of islanded microgrids with meshed topology," IEEE Transactions on Smart Grid, vol. 6, no. 3, pp. 1176-1184, 2015.

[21] Distribution Test Feeders. IEEE PES distribution system analysis subcommittee, 2011, available: http:/www.ewh.ieee.org/soc/pes/dsacom/ testfeeders/index.html.

[22] M. Tucci, A. Floriduz, S. Riverso, and G. Ferrari-Trecate, "Plug-andplay control of AC islanded microgrids with general topology," in 2016 European Control Conference (ECC), June 2016, pp. 1493-1500.

[23] R. H. Park, "Two-reaction theory of synchronous machines generalized method of analysis-part I," Transactions of the American Institute of Electrical Engineers, vol. 48, no. 3, pp. 716-727, 1929.

[24] R. N. Bracewell, The Fourier transform and its applications. McGrawHill New York, 1986, vol. 31999.

[25] B. Bollobás, Modern graph theory, ser. Graduate Texts in Mathematics. Springer Science \& Business Media, 1998, vol. 184.

[26] S. Y. Caliskan and P. Tabuada, "Kron reduction of generalized electrical networks," arXiv preprint arXiv:1207.0563, 2012.

[27] C. A. Desoer and E. S. Kuh, Basic circuit theory. Tata McGraw-Hill Education, 1984.

[28] J. Schiffer, "Stability and power sharing in microgrids," Ph.D. thesis, TU Berlin, Germany, 2015, available: https:/opus4.kobv.de/opus4-tuberlin/ files/6940/schiffer_johannes.pdf.

[29] D. Vakman, "On the analytic signal, the Teager-Kaiser energy algorithm, and other methods for defining amplitude and frequency," IEEE Transactions on Signal Processing, vol. 44, no. 4, pp. 791-797, Apr 1996.

[30] P. Penfield, R. Spence, and S. Duinker, "A generalized form of Tellegen's theorem," IEEE Transactions on Circuit Theory, vol. 17, no. 3, pp. 302 305, Aug 1970.

[31] M. Tucci, A. Floriduz, S. Riverso, and G. Ferrari-Trecate, "Kron reduction methods for plug-and-play control of AC islanded microgrids with arbitrary topology," Tech. Rep., 2015. [Online]. Available: https://arxiv.org/abs/1510.07873

[32] H. Akagi, E. H. Watanabe, and M. Aredes, Instantaneous power theory and applications to power conditioning. John Wiley \& Sons, 2007.

[33] M. Babazadeh and H. R. Karimi, "A robust two-degree-of-freedom control strategy for an islanded microgrid," IEEE Transactions on Power Delivery, vol. 28, no. 3, pp. 1339-1347, 2013.

[34] S. Bolognani and S. Zampieri, "A distributed control strategy for reactive power compensation in smart microgrids," IEEE Transactions on Automatic Control, vol. 58, no. 11, pp. 2818-2833, 2013.

[35] "IEEE recommended practice for monitoring electric power quality," IEEE Std 1159-2009 (Revision of IEEE Std 1159-1995), pp. c1-81, June 2009.

[36] K. J. Åström and T. Hägglund, Advanced PID control. ISA-The Instrumentation, Systems, and Automation Society; Research Triangle Park, NC 27709, 2006.

[37] M. Tucci, S. Riverso, J. C. Vasquez, J. M. Guerrero, and G. FerrariTrecate, "A decentralized scalable approach to voltage control of DC islanded microgrids," IEEE Transactions on Control Systems Technology, vol. 24, no. 6, pp. 1965-1979, Nov 2016. 